\title{
Meandering rivers: How important is lateral variability for species persistence?
}

\author{
Yu Jin* Frithjof Lutscher†, Yuan Pei ${ }^{\ddagger}$
}

\begin{abstract}
Models for population dynamics in rivers and streams have highlighted the importance of spatial and temporal variations for population persistence. We present a novel model that considers the longitudinal variation as introduced by the sinuosity of a meandering river where a main channel is laterally extended to point bars in bends. These regions offer different habitat conditions for aquatic populations and therefore may enhance population persistence. Our model is a nonstandard reaction-advection-diffusion model where the domain of definition consists of the real line (representing the main channel) with periodically added intervals (representing the point bars). We give an existence and uniqueness proof for solutions of the equations. We then study population persistence as the (in-) stability of the trivial solution and population spread as the minimal wave speed of traveling periodic waves. We conduct a sensitivity analysis to highlight the importance of each parameter on the model outcome. We find that sinuosity can enhance species persistence.
\end{abstract}

\section{Keywords}

Meandering Rivers; Point Bars; Drift Paradox; Population Persistence; Eigenvalue Problem; Minimal Wave Speeds

\section{Introduction}

The question of how a biological population manages to persist in a stretch of a river, despite being subject to wash-out from downstream flow, was first raised in the biological literature by Müller [1954] and later termed the 'drift paradox'. It seems clear that upstream flight of stream insects with winged adult stages could off-set downstream drift of early life stages [Hershey et al., 1993, Müller, 1982, Vasilyeva et al., 2016]; see Lutscher et al. [2010] for more details and

\footnotetext{
*Department of Mathematics, University of Nebraska-Lincoln, Lincoln, NE, 68588, USA. Email: yjin6@unl.edu

${ }^{\dagger}$ Department of Mathematics and Statistics, Department of Biology, University of Ottawa, Ottawa, ON, K1N6N5, Canada. Email: flutsche@uottawa.ca

${ }^{\ddagger}$ Department of Mathematics, University of Nebraska-Lincoln, Lincoln, NE, 68588, USA. Email: ypei4@unl.edu
} 
references on this topic. However, many species do not have such life stages and still persist locally in streams and rivers, for example algae.

After some initial simulation-based studies [Anholt, 1995], the question of population persistence in riverine environments was cast into a more rigorous modelling framework by Speirs and Gurney [2001]. These authors derived a reaction-advection-diffusion equation for the dynamics of a population and calculated the 'critical domain size', i.e. the minimal length of the domain for which the trivial solution is unstable. They found that simple diffusion can promote population persistence, but - given realistic parameter values for several organisms in real streams and rivers - is unlikely to do so in practice. Including more realistic, depth-dependent flow profiles the same authors then showed numerically that realistic magnitudes of diffusion can ensure population persistence.

The work by Speirs and Gurney [2001] inspired a number of authors to explore other aspects of population dynamics in rivers, for example by simplifying the representation of depthdependent flow profiles into two compartments [Pachepsky et al., 2005, Lutscher et al., 2005], introducing longitudinal variability in the form of pool-riffle sequences [Lutscher et al., 2006, Jin et al., 2014] or gradients [Lutscher et al., 2007, Lam et al., 2016], or by considering temporal variability [Lutscher and Seo, 2011, Seo and Lutscher, 2011, Jin and Lewis, 2011] and stochastic effects [Kolpas and Nisbet, 2010, Pasour and Ellner, 2010, Samia and Lutscher, 2012]. One recurring insight is that variability, in space or time, can often facilitate population persistence in rivers.

Almost all these studies implicitly assume that the stream or river is a straight channel. The separation of flow and benthic compartments captures some aspect of the horizontally and vertically varying flow profile, whereas the periodically alternating channel cross-section captures a pool-riffle structure, but still assumes a well-mixed population in each cross section. Natural rivers, on the contrary, rarely flow straight but show longitudinally meandering patterns as in Figure 1. The physical processes behind the emergence of meanders are reasonably well understood, but their effects and importance for population-dynamic processes has not yet been explored. Depending on the shape of the river bed, a variety of different flow-speed profiles can arise in a meander. A typical profile shows deeper and faster water at the outside of a bend ('bluff') and shallower, slower water on the inside ('point bar'). At the same time, there is a helicoidal flow that mixes water laterally; see Figure 1 and also e.g., [?]. While there seems to be no truly predictive theory of flow profiles in a real meandering river (Peter Steffler, personal communication), we use this scenario for our model. There is good agreement with at least one numerically computed flow field in a simplified sinusoidal model river [Jin et al., 2014].

In analogy with the drift-benthic division [Pachepsky et al., 2005], we conceptually split the meandering river into two compartments: a 'main channel' with faster flow and a shallower region with slower flow, corresponding to the inner part of a bend. We refer to the shallower part as 'point bar', noting that we think of the submerged part only while the term typically refers to the exposed part as well. We conceptualize our river schematically in Figure 2. In between bends, where the river runs relatively straight, there is no shallow component; in the 

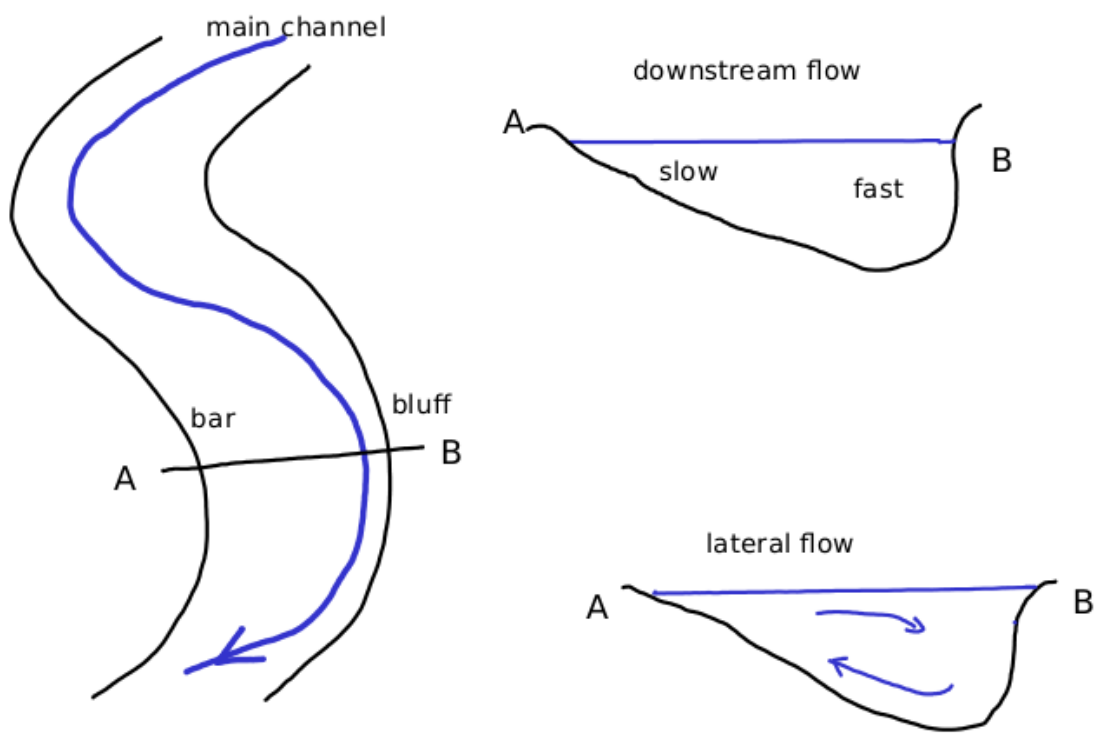

Figure 1: Illustration of a meandering river course. The top view (left) shows the line of the main channel within the river bed and the location of the point bar and bluff in a bend. The two cross sections show the longitudinal (top right) and lateral (bottom right) flow characteristics in a bend.

bends we have the point bars. To make this model somewhat analytically tractable, we assume that the river meanders periodically. Appropriate interface conditions describe the part of the population entering the shallow component versus the main channel.

In Section 2, we construct our simplified meandering river models for two scenarios, and we derive a reaction-advection-diffusion system for a population in the river. We then study and illustrate persistence conditions for a single logistically growing population on a (periodic) unbounded domain and on a bounded domain in Section 3. In Section 4, we derive minimal wave speeds of spatially periodic traveling waves in the upstream and downstream directions. In both cases, we illustrate the influences of different biotic and abiotic parameters on population persistence, measured in terms of instability of the trivial state (Section 3) or in terms of ability to spread upstream (Section 4). We present some results on the existence and regularity of solutions of our novel model in the appendix.

\section{Model}

We begin with a detailed derivation of the model equations that we study later. Our central idea is to split the continuously varying cross-sectional profile into two compartments: one for the main channel and one for the point bar; see Figure 2. While this simplification may seem too coarse to capture the details of helicoidal flow, we note that a similar two-compartment simplification of the water and hyporheic zone provides a surprisingly accurate description of stream flow [Bencala and Walters, 1983]. We consider two slightly different scenarios as indicated 
in the figure.

\section{$2.1 \quad$ River scenarios}

We represent the main channel of the river as a continuous domain and the point bars as periodically added components, see Figure 2. In view of the analytical tools and techniques available, we assume periodically spaced point bars of period $L$. In each period, the stream consists of a main channel and a point bar, located at $\left(k L, k L+L_{1}\right]$, and the main channel only at $\left(k L+L_{1},(k+1) L\right]$, with $k \in \mathbb{Z}$ and $L_{1}<L$. We denote the cross-sectional areas of the main channel and the point bar in $\left(k L, k L+L_{1}\right]$, as $A_{1}$ and $A_{w}$, respectively, whereas the crosssectional area of the main channel in $\left(k L+L_{1},(k+1) L\right]$ is denoted by $A_{2}$. The corresponding flow velocities are denoted by $v_{1}, v_{w}$ and $v_{2}$. To unify notation, we write $A_{u}$ for the cross-sectional area of the main channel, so that $A_{u}=A_{1}$ or $A_{u}=A_{2}$, depending on which section of the main channel we have.

Now we assume that there is no lateral water exchange outside of the river bed so that the discharge remains constant downstream. This assumption gives the hydrological condition

$$
A_{1} v_{1}+A_{w} v_{w}=A_{2} v_{2}
$$

To reduce the number of parameters in our model a little further, we consider two scenarios for how the cross-sectional areas along the river are related, see Figure 2. In the first scenario, the cross-sectional area of the river does not change over one period. The point bar is 'carved out' from the main channel. In the second scenario, the cross-sectional area of the main channel is fixed and the point bar gives additional habitat. Because of the constant discharge assumption in (1), the relationship between cross-sectional areas has implications for the flow speeds in the different sections of the river. The exact relations are as follows.

(S1) The overall cross-section is constant: $A_{1}+A_{w}=A_{2}$. Then the flow speeds satisfy

$$
v_{2}=\frac{v_{1} A_{1}+v_{w} A_{w}}{A_{1}+A_{w}}
$$

We refer to this case as constant cross-section (CCS).

(S2) The cross section of the main channel is constant: $A_{1}=A_{2}$. Then the flow speeds satisfy

$$
v_{2}=\frac{v_{1} A_{1}+v_{w} A_{w}}{A_{2}}=v_{1}+\frac{A_{w}}{A_{1}} v_{w}
$$

We refer to this case as constant main channel (CMC).

\subsection{Population dynamics}

We denote by $u(x, t)$ and $w(x, t)$ the densities of a population (unit: individuals per volume) in the main channel and in the point bar, respectively. Within each of these compartments, 


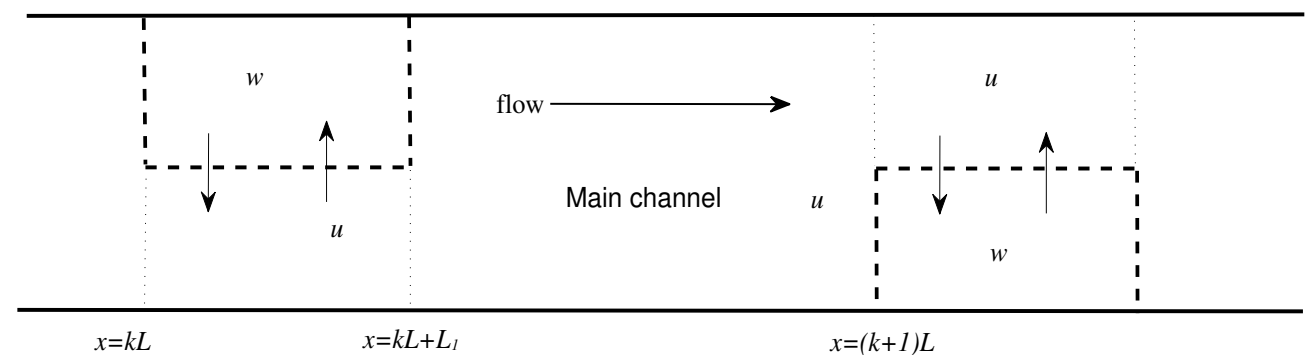

Scenaio 2: CMC

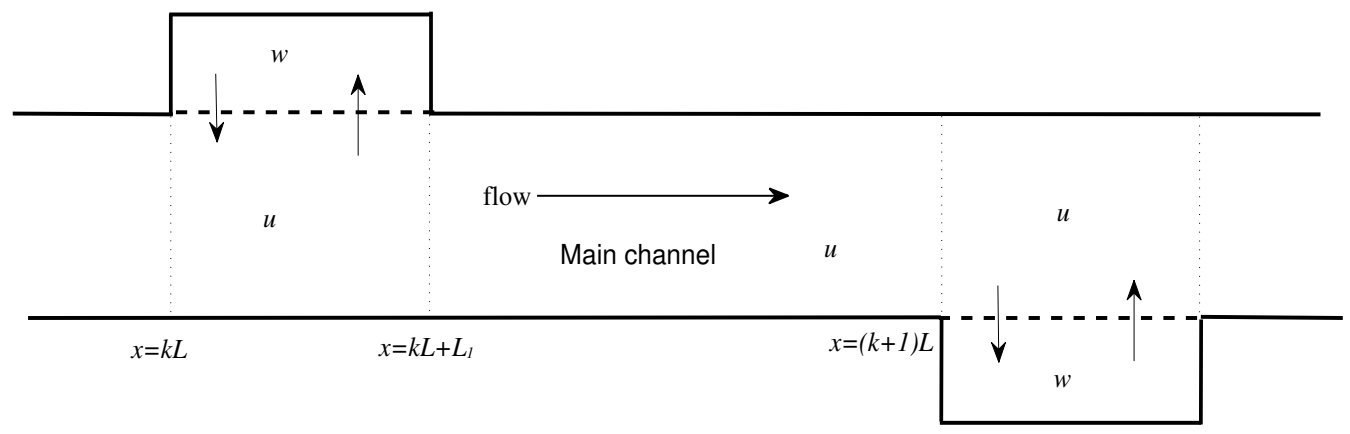

Figure 2: Simplified schematic for a periodically meandering river. Scenario 1 (CCS): the river channel has a constant cross-sectional area. The point bar (dashed rectangle) reduces the cross section of the main channel. Scenario 2 (CMC): the main channel has a constant cross section and the point bar (separated by the dashed lines) adds cross-sectional area. Variables $u$ and $w$ will denote the population densities in the respective regions. Parameter $L_{1}$ denotes the length of the point bar and $L$ the period of the river. The horizontal arrow indicates flow direction, the vertical arrows indicate the lateral exchange between main channel and point bar.

we assume that the population is laterally well mixed, so that $u$ and $w$ are functions of time $t$ and downstream distance $x$. Diffusion rates are $D_{u}$ and $D_{w}$, respectively. We denote by $f$ the per-capita production rate in the main channel, and in the point bar by $g$. These functions can vary with longitudinal distance. Function $g$ is zero when $x \in\left(k L+L_{1},(k+1) L\right]$.

Writing the mass balance for the population gives the model equations

$$
\left\{\begin{aligned}
\left(A_{u} u\right)_{t} & =\left(D_{u} A_{u} u_{x}\right)_{x}-\left(v_{u} A_{u} u\right)_{x}+\alpha_{w} A_{w} w-\alpha_{u} A_{u} u+A_{u} f(x, u) u, \\
\left(A_{w} w\right)_{t} & =\left(D_{w} A_{w} w_{x}\right)_{x}-\left(v_{w} A_{w} w\right)_{x}+\alpha_{u} A_{u} u-\alpha_{w} A_{w} w+A_{w} g(x, w) w
\end{aligned}\right.
$$

where subscripts $t$ and $x$ denote partial derivatives. Rate parameters $\alpha_{u}$ and $\alpha_{w}$ are the lateral exchange rates induced by the helicoidal flow between main channel and point bar where the latter exists. Otherwise, these coefficients are zero. To preserve the volume of water in the main 
channel versus the point bar, we require the following condition for the exchange flows

$$
\alpha_{w} A_{w}=\alpha_{u} A_{u}
$$

We shall always assume the following about the coefficient functions in model (4).

(A1) All parameters are piecewise continuous, positive, $L$-periodic functions of downstream distance. Parameters are independent of time.

(A2) Growth functions $f(x, u), g(x, v)$ are piecewise continuous and $L$-periodic in $x$ and differentiable with respect to their second argument.

(A3) For each $x$, the growth function $f(x, u)$ is strictly decreasing in $u \in(0, \infty)$ with $f(x, 0)<$ $\infty$, and there exists some $K>0$ such that $f(x, u) \leq 0$ for all $u \geq K$. The growth function $g$ satisfies the same conditions.

At this point, we follow previous authors who studied population dynamics in periodic environments and assume that coefficient functions are piecewise constant (e.g. Shigesada et al. [1986]). Then we write index 1 to denote the value in sections with point bar and index 2 for sections without. Specifically, for the $u$-equation we write $A_{1}, v_{1}, D_{1}, f_{1}, \alpha_{u}$ for $x \in\left(k L, k L+L_{1}\right)$ and $A_{2}, v_{2}, D_{2}, f_{2}$ for $x \in\left(k L+L_{1},(k+1) L\right)$, and for the $w$-equation we write $A_{w}, v_{w}, D_{w}, \alpha_{w}$ for $x \in\left(k L, k L+L_{1}\right)$.

With these assumptions, we can divide by the cross-sectional areas and write our system as

$$
\left\{\begin{array}{lc}
u_{t}=D_{1} u_{x x}-v_{1} u_{x}+\alpha_{u}(w-u)+f_{1}(u) u, & x \in\left(k L, k L+L_{1}\right), \\
w_{t}=D_{w} w_{x x}-v_{w} w_{x}+\alpha_{w}(u-w)+g(w) w, & x \in\left(k L, k L+L_{1}\right), \\
u_{t}=D_{2} u_{x x}-v_{2} u_{x}+f_{2}(u) u . & x \in\left(k L+L_{1},(k+1) L\right),
\end{array}\right.
$$

with initial distributions $u^{0}$ on $[k L,(k+1) L]$ and $w^{0}$ on $\left[k L, k L+L_{1}\right]$.

Finally, we need to impose conditions at the interfaces in the river, i.e. the locations where point bars begin and end. Following Ramirez [2012], Sarhad et al. [2014], we require the concentration of the population to be continuous at the interior interfaces $y_{k}^{0}=k L$ and $y_{k}^{1}=k L+L_{1}$, which requires

$$
\begin{aligned}
& \lim _{x \uparrow y_{k}^{0}} u(x, t)=\lim _{x \downarrow y_{k}^{0}} u(x, t)=\lim _{x \downarrow y_{k}^{0}} w(x, t), t>0, \\
& \lim _{x \uparrow y_{k}^{1}} u(x, t)=\lim _{x \downarrow y_{k}^{1}} u(x, t)=\lim _{x \uparrow y_{k}^{1}} w(x, t), t>0 .
\end{aligned}
$$

The total population flux should satisfy a conservation law: at $y_{k}^{1}$, the fluxes out of the main channel and the point bar combined should equal the flux into the main channel compartment immediately downstream, and vice versa at $y_{k}^{0}$. Hence, we write

$$
\lim _{x \uparrow y_{k}^{1}}\left[\left(A_{1} D_{1} u_{x}-v_{1} A_{1} u\right)+\left(A_{w} D_{w} w_{x}-v_{w} A_{w} w\right)\right](x, t)=\lim _{x \downarrow y_{k}^{1}}\left(A_{2} D_{2} u_{x}-v_{2} A_{2} u\right)(x, t), t>0
$$


and similarly for $y_{k}^{0}$. Since the densities are continuous by (7), the constant discharge assumption (1) reduces the flux conditions (8) to

$$
\begin{aligned}
& \lim _{x \uparrow y_{k}^{1}}\left[A_{1} D_{1} u_{x}+A_{w} D_{w} w_{x}\right](x, t)=\lim _{x \downarrow y_{k}^{1}}\left(A_{2} D_{2} u_{x}\right)(x, t), t>0, \\
& \lim _{x \downarrow y_{k}^{0}}\left[A_{1} D_{1} u_{x}+A_{w} D_{w} w_{x}\right](x, t)=\lim _{x \uparrow y_{k}^{0}}\left(A_{2} D_{2} u_{x}\right)(x, t), t>0 .
\end{aligned}
$$

On a bounded domain, say $[0, n L]$ for some positive integer $n$, we require additional conditions at the boundary points $x=0$ and $x=n L$. A number of conditions have been suggested in such situations. For example no-flux conditions are often imposed upstream, whereas 'free-flow' conditions [Ballyk et al., 1998] or hostile conditions [Speirs and Gurney, 2001] can be imposed downstream. In keeping with the classical critical patch size problem, hostile conditions can also be imposed upstream. In our context these conditions read

$$
\begin{array}{llll}
\text { upstream: } & \left(A_{1} D_{1} u_{x}-v_{1} A_{1} u+A_{w} D_{w} w_{x}-v_{w} A_{w} w\right)(0, t)=0 & \text { or } & u(0, t)=w(0, t)=0 ; \\
\text { downstream: } & u_{x}(n L, t)=0 & \text { or } & u(n L, t)=0,
\end{array}
$$

for all $t>0$. Lou and Lutscher [2014] provide a detailed derivation and discussion of these conditions.

The following theorem provides the well-posedness for system (6) on a periodic bounded domain. The proof is provided in Appendix A, where we also clarify the terminology.

Theorem 2.1 Assume that (A1), (A2), and (A3) hold. Suppose that $u_{0}, w_{0} \in L^{2}$ with $u_{0}, w_{0} \geq$ 0 . For any $T>0$, there exists a unique weak solution of (6) under the interface conditions (7) and (9), and hostile upstream and downstream boundary conditions over a bounded periodic domain $[0, n L]$ for any positive integer $n$. Moreover, if $0 \leq u_{0}, w_{0} \in H^{1}$, then the solution is regular, that is, $u, w \in L^{\infty}\left(H^{1} ;[0, T]\right) \cap L^{2}\left(H^{2} ;[0, T]\right)$.

Remark 2.1 The proof of the above theorem carries over with minor modifications to the case of an unbounded periodic habitat. Also, one can show that solutions are actually twice differentiable everywhere except at the interface points.

\section{Persistence conditions}

In this section, we derive conditions for population persistence in an unbounded domain and in a bounded domain. Since assumption (A3) on the growth function excludes an Allee effect, population persistence is guaranteed if the population can grow at low density, or, in other words, if the trivial steady state is unstable (see e.g., Jin and Lewis [2011], Lutscher et al. [2005, 2006]). Therefore, we linearize the equations in (6) at low density and consider the corresponding 
eigenvalue problem. The linearization is given by

$$
\left\{\begin{array}{l}
u_{t}=D_{1} u_{x x}-v_{1} u_{x}+\alpha_{u}(w-u)+r_{1} u, \quad x \in\left(k L, k L+L_{1}\right), \\
w_{t}=D_{w} w_{x x}-v_{w} w_{x}+\alpha_{w}(u-w)+r_{w} w, \quad x \in\left(k L, k L+L_{1}\right), \\
u_{t}=D_{2} u_{x x}-v_{2} u_{x}+r_{2} u . \quad x \in\left(k L+L_{1},(k+1) L\right),
\end{array}\right.
$$

with the interface conditions (7) and (9). Here $r_{i}=f_{i}^{\prime}(0)$ and $r_{w}=g^{\prime}(0)$.

We derive expressions for the persistence boundary, the set in parameter space where the dominant eigenvalue is equal to zero. This set delineates the boundary between population persistence and extinction. We split the calculations into two cases, one on the unbounded domain and one on a bounded domain.

\subsection{Persistence on the unbounded domain}

To study persistence of the population on the unbounded domain, we need to consider only the equivalent periodic domain of length $L$ [Shigesada et al., 1986, Lutscher et al., 2006] with interface conditions at $x=L_{1}$ as above and periodic boundary conditions at $x=0$ and $x=L$ :

$$
\begin{aligned}
& \lim _{x \downarrow 0} u(x, t)=\lim _{x \downarrow 0} w(x, t)=\lim _{x \uparrow L} u(x, t), \\
& \lim _{x \downarrow 0}\left[A_{1} D_{1} u_{x}+A_{w} D_{w} w_{x}\right](x, t)=\lim _{x \uparrow L}\left(A_{2} D_{2} u_{x}\right)(x, t), \\
& \lim _{x \uparrow L_{1}} u(x, t)=\lim _{x \uparrow L_{1}} w(x, t)=\lim _{x \downarrow L_{1}} u(x, t), \\
& \lim _{x \uparrow L_{1}}\left[A_{1} D_{1} u_{x}+A_{w} D_{w} w_{x}\right]=\lim _{x \downarrow L_{1}}\left(A_{2} D_{2} u_{x}\right)
\end{aligned}
$$

for all $t>0$.

\subsubsection{Persistence boundary}

With the ansatz $u(x, t)=\phi(t) \psi(x)$ and $w(x, t)=\phi_{w}(t) \psi_{w}(x)$, the linearized system (11) on $(0, L)$ becomes

$$
\left\{\begin{array}{l}
\frac{\phi^{\prime}}{\phi}=D_{1} \frac{\psi^{\prime \prime}}{\psi}-v_{1} \frac{\psi^{\prime}}{\psi}+\alpha_{u} \frac{\phi_{w} \psi_{w}}{\phi \psi}-\alpha_{u}+r_{1}, \quad x \in\left(0, L_{1}\right) \\
\frac{\phi_{w}^{\prime}}{\phi_{w}}=D_{w} \frac{\psi_{w}^{\prime \prime}}{\psi_{w}}-v_{w} \frac{\psi_{w}^{\prime}}{\psi_{w}}+\alpha_{w} \frac{\phi \psi}{\phi_{w} \psi_{w}}-\alpha_{w}+r_{w}, \quad x \in\left(0, L_{1}\right) \\
\frac{\phi^{\prime}}{\phi}=D_{2} \frac{\psi^{\prime \prime}}{\psi}-v_{2} \frac{\psi^{\prime}}{\psi}+r_{2}, \quad x \in\left(L_{1}, L\right),
\end{array}\right.
$$

with corresponding interface and boundary conditions. These equations imply that $\phi^{\prime} / \phi=-s$ is a constant and that $\phi_{w} / \phi$ is a constant as well. Without loss of generality, we can set $\phi_{w}=\phi$ and incorporate the constant into $\psi_{w}$. For $s>0$, the population declines exponentially; for $s<0$ it grows. To find the persistence boundary in parameter space for this population, we set $s=0$, 
and solve the second-order system

$$
\left\{\begin{array}{l}
D_{1} \psi^{\prime \prime}-v_{1} \psi^{\prime}+\alpha_{u} \psi_{w}+\left(r_{1}-\alpha_{u}\right) \psi=0, \quad x \in\left(0, L_{1}\right) \\
D_{w} \psi_{w}^{\prime \prime}-v_{w} \psi_{w}^{\prime}+\alpha_{w} \psi+\left(r_{w}-\alpha_{w}\right) \psi_{w}=0, \quad x \in\left(0, L_{1}\right) \\
D_{2} \psi^{\prime \prime}-v_{2} \psi^{\prime}+r_{2} \psi=0, \quad x \in\left(L_{1}, L\right)
\end{array}\right.
$$

with corresponding interface and boundary conditions. We solve the first of these equations for $\psi_{w}$ and get

$$
\psi_{w}=\frac{1}{\alpha_{u}}\left[-D_{1} \psi^{\prime \prime}+v_{1} \psi^{\prime}-\left(r_{1}-\alpha_{u}\right) \psi\right] .
$$

Substituting this expression into the second equation in (14) gives the fourth-order system

$$
\left\{\begin{array}{cc}
D_{1} D_{w} \psi^{(4)}-\left(D_{1} v_{w}+D_{w} v_{1}\right) \psi^{(3)}+\left[D_{w}\left(r_{1}-\alpha_{u}\right)+v_{1} v_{w}+D_{1}\left(r_{w}-\alpha_{w}\right)\right] \psi^{\prime \prime} & \\
-\left[v_{w}\left(r_{1}-\alpha_{u}\right)+v_{1}\left(r_{w}-\alpha_{w}\right)\right] \psi^{\prime}+\left[\alpha_{u} \alpha_{w}+\left(r_{1}-\alpha_{u}\right)\left(r_{w}-\alpha_{w}\right)\right] \psi=0, & x \in\left(0, L_{1}\right) \\
D_{2} \psi^{\prime \prime}-v_{2} \psi^{\prime}+r_{2} \psi=0, & x \in\left(L_{1}, L\right) .
\end{array}\right.
$$

Denoting the solutions of the quadratic characteristic equation on $\left(0, L_{1}\right)$ by $\lambda_{1}, \ldots, \lambda_{4}$, we can write

$$
\psi(x)=\sum_{i=1}^{4} S_{i} e^{\lambda_{i} x}
$$

assuming that we are in the generic case of no repeated roots. On the interval $\left(L_{1}, L\right)$, we get an explicit solution for the characteristic equation and write

$$
\psi(x)=S_{5} e^{-\lambda_{5}(L-x)}+S_{6} e^{-\lambda_{6}(L-x)}, \quad \lambda_{5,6}=\frac{v_{2} \pm \sqrt{v_{2}^{2}-4 D_{2} r_{2}}}{2 D_{2}} .
$$

From (15) we obtain the expression

$$
\psi_{w}(x)=\sum_{i=1}^{4} \frac{1}{\alpha_{u}}\left[-D_{1} \lambda_{i}^{2}+v_{1} \lambda_{i}-\left(r_{1}-\alpha_{u}\right)\right] S_{i} e^{\lambda_{i} x}=: \sum_{i=1}^{4} \theta_{i} S_{i} e^{\lambda_{i} x}
$$

Now, we use the matching conditions at $x=0, L_{1}$ and $L$ to find conditions for the existence of a nonzero solution for constants $S_{1}, \ldots, S_{6}$. After some algebra, these conditions are equivalent to the following matrix having zero determinant:

$$
\mathbf{M}=\left[\begin{array}{cccccc}
1 & 1 & 1 & 1 & -1 & -1 \\
e^{\lambda_{1} L_{1}} & e^{\lambda_{2} L_{1}} & e^{\lambda_{3} L_{1}} & e^{\lambda_{4} L_{1}} & -e^{-\lambda_{5} L_{2}} & -e^{-\lambda_{6} L_{2}} \\
\gamma_{1} \lambda_{1} e^{\lambda_{1} L_{1}} & \gamma_{2} \lambda_{2} e^{\lambda_{2} L_{1}} & \gamma_{3} \lambda_{3} e^{\lambda_{3} L_{1}} & \gamma_{4} \lambda_{4} e^{\lambda_{4} L_{1}} & -A_{2} D_{2} \lambda_{5} e^{-\lambda_{5} L_{2}} & -A_{2} D_{2} \lambda_{6} e^{-\lambda_{6} L_{2}} \\
\gamma_{1} \lambda_{1} & \gamma_{2} \lambda_{2} & \gamma_{3} \lambda_{3} & \gamma_{4} \lambda_{4} & -A_{2} D_{2} \lambda_{5} & -A_{2} D_{2} \lambda_{6} \\
1-\theta_{1} & 1-\theta_{2} & 1-\theta_{3} & 1-\theta_{4} & 0 & 0 \\
\left(1-\theta_{1}\right) e^{\lambda_{1} L_{1}} & \left(1-\theta_{2}\right) e^{\lambda_{2} L_{1}} & \left(1-\theta_{3}\right) e^{\lambda_{3} L_{1}} & \left(1-\theta_{4}\right) e^{\lambda_{4} L_{1}} & 0 & 0
\end{array}\right]
$$


Here, we let $L_{2}=L-L_{1}$ and denoted $\gamma_{i}=\left(A_{1} D_{1}+A_{w} D_{w} \theta_{i}\right)$. In summary, we proved the following result.

Theorem 3.1 The stability boundary of the trivial steady state of (6) on the infinite domain under interface conditions (7) and (9) occurs where the determinant of the matrix $\mathbf{M}$ in (20) is zero.

Remark 3.1 In Sections 3 and 4, general solutions of ordinary differential equations (see e.g., (17) and (18)) are only given in the case where the corresponding characteristic equations have no repeated roots. However, in numerical simulations, we consider solutions in all possible cases where the corresponding characteristic equations have distinct or repeated, real or complex roots.

Calculation of the $6 \times 6$ determinant in (20) can be simplified somewhat by considering its block-structure. However, since the solutions $\lambda_{i}$ of the quartic have to be calculated numerically in any case, we do not attempt to find a more explicit formula of this determinant and instead proceed by solving for the persistence boundary computationally. To that end, we pick baseline parameter values and do a local sensitivity analysis, i.e. we report how the minimal size requirement depends on changes in the parameters. We illustrate the results, using the two cases CCS and $\mathrm{CMC}$ as described in the previous section.

\subsubsection{Unfavorable main channel}

When the main channel is unfavorable for the species, we have $r_{1}, r_{2}<0$. Clearly, the species cannot persist in the absence of a region of positive growth rate. Hence, we explore how large a point bar with $r_{w}>0$ is required for the species to persist and how sensitive this requirement is to changes in the other parameters. Roughly, the size of the point bar should increase as the sinuosity of the river increases. Adding point bars with $A_{w}>0$ while keeping the main-channel cross-section constant $\left(A_{1}=A_{2}\right)$ corresponds to the CMC case and implies that flow speed $v_{2}$ will increase compared to the case when $A_{w}=0$ as in relation (3). While the addition of a region with positive growth rate should make persistence more likely, the increased flow speed will counteract this effect somewhat.

When we choose baseline parameters as in Table 1, the minimal point-bar cross-section that allows for persistence is $A_{w}^{*}=0.4657$, or $46.57 \%$ of the main channel cross section. We illustrate the sensitivity of this critical value to changes in the other parameters. Specifically, we increased each parameter separately by $10 \%$ and by $50 \%$ and calculated the resulting minimum area $\widehat{A}_{w}^{*}$.

The table contains the ratio $\widehat{A}_{w}^{*} / A_{w}^{*}$ in each case. We scaled space to set $L_{1}=1$ and time to set $r_{w}=1$. In addition, we used the simplifying assumption that the growth rate in the main channel is constant $\left(r_{1}=r_{2}<0\right)$ and the CMC condition. We ranked parameters according to their influence.

The critical value $A_{w}^{*}$ is most sensitive to changes in the lateral exchange rate $\alpha_{u}$. Increasing $\alpha_{u}$ increases lateral movement of individuals and decreases residence time in the point bar where 


\begin{tabular}{|c|c|c|c|c|c|c|c|c|c|}
\hline parameter $(p)$ & $\alpha_{u}$ & $A_{1}$ & $v_{1}$ & $D_{w}$ & $\left|r_{1}\right|$ & $v_{w}$ & $L_{2}$ & $D_{2}$ & $D_{1}$ \\
\hline \hline baseline value & 0.4 & 1 & 1 & 1 & 0.1 & 0.1 & 1 & 1 & 1 \\
\hline \hline$\widehat{A}_{w}^{*} / A_{w}^{*}$ as $p \uparrow 10 \%$ & 1.1165 & 1.100 & 1.0750 & 1.0705 & 1.0147 & 1.0053 & 1 & 1 & 0.9141 \\
\hline$\widehat{A}_{w}^{*} / A_{w}^{*}$ as $p \uparrow 50 \%$ & 1.7058 & 1.500 & 1.3054 & 1.3574 & 1.0837 & 1.0271 & 1 & 1 & 0.7502 \\
\hline
\end{tabular}

Table 1: Illustration of the population persistence condition from Theorem 3.1. We present the sensitivity of the minimum point-bar area to changes in other model parameters in the case of an unfavorable, constant cross-section main channel (CMC). Model parameters are in the first row, their default parameter values in the second. The resulting minimal point-bar cross-section is $A_{w}^{*}=0.4657$. The third and fourth row give the ratio $\widehat{A}_{w}^{*} / A_{w}^{*}$ resulting from a $10 \%(50 \%)$ increase in the parameter's value.

the population can grow. Hence, a larger point bar is required for persistence. Cross-sectional area of the main channel, flow speed in the main channel or in the point bar, population loss rate and, noticeably, diffusion in the point bar, all increase the required critical value of $A_{w}^{*}$. The only parameter that is clearly negatively correlated with $A_{w}^{*}$ is the diffusion rate in the main channel. Diffusion in the main channel allows individuals to move upstream against the flow and thereby increases the likelihood of persistence so that a smaller point bar suffices.

\subsubsection{Source-sink main channel}

When the main channel consists of alternating sections, favorable sources $\left(r_{1}>0\right)$ and unfavorable sinks $\left(r_{2}<0\right)$, population persistence depends on the balance of a number of factors [Lutscher et al., 2006]. For example, the population cannot persist if the favorable patches are too small or the growth rate there is not high enough. Here, we consider the scenario that source patches in the main channel occur in bends where there is also a favorable point bar and sinks occur in the straighter connections between two bends where there is no point bar. This scenario is reminiscent of the pool-riffle setting found in many streams. Assuming that a population cannot persist in the absence of the slow-flowing environment of the point bar, we ask which portion, in terms of cross-sectional area, of the favorable patches would have to be a point bar so that persistence becomes possible. As above, stronger sinuosity of the river would correspond to larger point bars.

This scenario corresponds to the CCS set-up from Figure 2. If we fix the flow speed in the point bar, flow speed $v_{1}$ will increase as the size of the point bar increases. Again, there is some trade-off since while the low flow rate in the favorable point bar helps the population to persist, the main-channel part of the favorable section becomes less favorable since the flow speed there increases. As in the previous case, we establish a critical value for the cross-section of the point bar, $A_{w}^{*}$, and then ask by how much this value changes if parameters change. We summarize the results in Table 2 .

Only three parameters are positively correlated with the minimal required $A_{w}^{*}$. The strongest sensitivity is with respect to the cross-sectional area in the sink-section of the main channel, 


\begin{tabular}{|c|c|c|c|c|c|c|c|c|c|}
\hline parameter $(p)$ & $A_{2}$ & $\alpha_{u}$ & $v_{2}$ & $D_{2}$ & $\left|r_{2}\right|$ & $L_{2}$ & $v_{w}$ & $D_{w}$ & $D_{1}$ \\
\hline \hline baseline value & 1 & 0.5 & 1 & 1 & 2 & 5 & 0.1 & 1 & 1 \\
\hline \hline$\widehat{A}_{w}^{*} / A_{w}^{*}$ as $p \uparrow 10 \%$ & 1.100 & 1.0771 & 1.0483 & 1 & 1.000 & 1.000 & 0.9965 & 0.9955 & 0.9857 \\
\hline$\widehat{A}_{w}^{*} / A_{w}^{*}$ as $p \uparrow 50 \%$ & 1.500 & 1.3967 & 1.3584 & 1.000 & 1.000 & 1.000 & 0.9830 & 0.9801 & 0.9574 \\
\hline
\end{tabular}

Table 2: Illustration of the population persistence condition from Theorem 3.1. We present the sensitivity of the minimum point-bar area to changes in other model parameters in the case of a source-sink main channel and constant total cross-section (CCS). Model parameters are in the first row, their default parameter values in the second. The third and fourth row give the ratio $\widehat{A}_{w}^{*} / A_{w}^{*}$ resulting from a $10 \%(50 \%)$ increase in the parameter's value, where $\widehat{A}_{w}^{*}$ is the critical cross-section of the point bar for the increased parameter value.

followed by lateral exchange rate and by the flow speed in the sink part. Increasing the diffusion rates in the source-part of the main channel or the point bar, or the flow speed in the point bar all decrease the required $A_{w}^{*}$. The case of $v_{w}$ is somewhat surprising since we expect the point bar to be most effective when flow is slow there. However, increasing $v_{w}$ increases also $v_{1}$, so that the main channel there becomes less favorable. Increasing diffusion will increase the flow into the sink part of the main channel and therefore requires a larger cross-sectional area of the point bar.

\subsection{Persistence on a bounded domain}

Since, in reality, rivers are finite, we would like to have persistence conditions on bounded domains as well. In principle, the steps to calculate the persistence boundary are the same as above, but in practice, the derivations are more complex because one cannot reduce the problem to a single period of the domain. Instead, we assume that the river consists of $n$ periodic patches and has length of $n L$, i.e. the river is represented by the interval $[0, n L]$. We choose zero-flux boundary conditions at the upstream end and free-flow boundary conditions at the downstream end. Lutscher et al. [2006] took a similar approach in the case of a pool-riffle model, that corresponds to considering only the main channel in our model.

We study the equations

$$
\left\{\begin{array}{l}
u_{t}=D_{1} u_{x x}-v_{1} u_{x}+\alpha_{u}(w-u)+r_{1} u, \quad x \in\left(k L, k L+L_{1}\right), \quad 0 \leq k<n-1, \\
w_{t}=D_{w} w_{x x}-v_{w} w_{x}+\alpha_{w}(u-w)+r_{w} w, \quad x \in\left(k L, k L+L_{1}\right), \quad 0 \leq k<n-1, \\
u_{t}=D_{2} u_{x x}-v_{2} u_{x}+r_{2} u . \quad x \in\left(k L+L_{1},(k+1) L\right), \quad 0 \leq k<n-1,
\end{array}\right.
$$

with boundary conditions

$$
\begin{aligned}
\left(A_{1} D_{1} u_{x}-v_{1} u+A_{w} D_{w} w_{x}-v_{w} w\right)(0, t) & =0, t>0, \\
u_{x}(n L, t) & =0, t>0,
\end{aligned}
$$

and the same interface conditions at $x=k L$ or $x=k L+L_{1}$ as in (7) and (9). 
Similarly as in Section 3.1, letting $u(x, t)=\phi(t) \psi(x)$ and $w(x, t)=\phi_{w}(t) \psi_{w}(x)$, we obtain $\phi^{\prime} / \phi=-s$ for some constant $s$ and set $\phi_{w}=\phi$ without loss of generality. The persistence boundary then corresponds to setting $s=0$. As in the previous section, we can turn the system of three second-order equations into a system of two equations, one of fourth order and one of second. Hence, we try to find solutions to the system

$$
\left\{\begin{array}{cc}
D_{1} D_{w} \psi^{(4)}-\left(D_{1} v_{w}+D_{w} v_{1}\right) \psi^{(3)}+\left[D_{w}\left(r_{1}-\alpha_{u}\right)+v_{1} v_{w}+D_{1}\left(r_{w}-\alpha_{w}\right)\right] \psi^{\prime \prime} \\
-\left[v_{w}\left(r_{1}-\alpha_{u}\right)+v_{1}\left(r_{w}-\alpha_{w}\right)\right] \psi^{\prime}+\left[\alpha_{u} \alpha_{w}+\left(r_{1}-\alpha_{u}\right)\left(r_{w}-\alpha_{w}\right)\right] \psi=0, \\
x \in\left(k L, k L+L_{1}\right), 0 \leq k<n-1, \\
D_{2} \psi^{\prime \prime}-v_{2} \psi^{\prime}+r_{2} \psi=0, & x \in\left(k L+L_{1},(k+1) L\right), \quad 0 \leq k<n-1,
\end{array}\right.
$$

with boundary and interface conditions (7), (9), (22).

We replace the single function on the entire domain $(0, n L)$ by a family of functions on the domain $(0, L)$ by rescaling the argument. We let $\psi_{m}(x-m L)=\psi(x)$ for $x \in\left[m L, m L+L_{1}\right)$ and $z_{m}(x-m L)=\psi(x)$ for $x \in\left[m L+L_{1},(m+1) L\right)$, for $m=0,1, \ldots, n-1$. Then functions $\psi_{m}$ satisfy

$$
\left\{\begin{array}{c}
D_{1} D_{w} \psi_{m}^{(4)}-\left(D_{1} v_{w}+D_{w} v_{1}\right) \psi_{m}^{(3)}+\left[D_{w}\left(r_{1}-\alpha_{u}\right)+v_{1} v_{w}+D_{1}\left(r_{w}-\alpha_{w}\right)\right] \psi_{m}^{\prime \prime} \\
-\left[v_{w}\left(r_{1}-\alpha_{u}\right)+v_{1}\left(r_{w}-\alpha_{w}\right)\right] \psi_{m}^{\prime}+\left[\alpha_{u} \alpha_{w}+\left(r_{1}-\alpha_{u}\right)\left(r_{w}-\alpha_{w}\right)\right] \psi_{m}=0
\end{array}\right.
$$

for $x \in\left(0, L_{1}\right)$ while $z_{m}$ satisfy

$$
D_{2} z_{m}^{\prime \prime}-v_{2} z_{m}^{\prime}+r_{2} z_{m}=0, \quad x \in\left(L_{1}, L\right)
$$

Similarly as in (17)-(19), we can write

$$
\psi_{m}(x)=\sum_{i=1}^{4} S_{m_{i}} e^{\lambda_{i} x}, \quad \psi_{w_{m}}(x)=: \sum_{i=1}^{4} \theta_{i} S_{m_{i}} e^{\lambda_{i} x}, \quad x \in\left(0, L_{1}\right),
$$

and

$$
z_{m}(x)=S_{m_{5}} e^{-\lambda_{5}(L-x)}+S_{m_{6}} e^{-\lambda_{6}(L-x)}, \quad x \in\left(L_{1}, L\right),
$$

where $\lambda_{i}$ and $\theta_{i}$ are given as in (17)-(19) and $S_{m_{i}}$ are free parameters. The matching conditions at the interface and boundary points give the conditions to determine parameters $S_{m_{i}}$. These conditions can be written explicitly as follows.

The upstream matching conditions for $m=0$ are

$$
\left\{\begin{array}{l}
\sum_{i=1}^{4} S_{0_{i}}\left(\left(A_{1} D_{1}+A_{w} D_{w} \theta_{i}\right) \lambda_{i}-v_{1}-v_{w} \theta_{i}\right)=0, \\
\sum_{i=1}^{4} S_{0_{i}}\left(1-\theta_{i}\right) e^{\lambda_{i} L_{1}}=0, \\
\sum_{i=1}^{4} S_{0_{i}}\left(1-\theta_{i}\right)=0, \\
\sum_{i=1}^{4} S_{0_{i}} e^{\lambda_{i} L_{1}}-S_{0_{5}} e^{-\lambda_{5} L_{2}}-S_{0_{6}} e^{-\lambda_{6} L_{2}}=0, \\
\sum_{i=1}^{4} \lambda_{i} e^{\lambda_{i} L_{1}} S_{0_{i}}\left(A_{1} D_{1}+A_{w} D_{w} \theta_{i}\right)-A_{2} D_{2}\left(S_{0_{5}} \lambda_{5} e^{-\lambda_{5} L_{2}}+S_{0_{6}} \lambda_{6} e^{-\lambda_{6} L_{2}}\right)=0 .
\end{array}\right.
$$


The interface matching conditions for $0<m<n-1$ are

$$
\left\{\begin{array}{l}
\sum_{i=1}^{4} \lambda_{i} S_{m_{i}}\left(A_{1} D_{1}+A_{w} D_{w} \theta_{i}\right)-A_{2} D_{2} \lambda_{5} S_{(m-1)_{5}}-A_{2} D_{2} \lambda_{6} S_{(m-1)_{6}}=0, \\
\sum_{i=1}^{4} S_{m_{i}}-S_{(m-1)_{5}}-S_{(m-1)_{6}}=0, \\
\sum_{i=1}^{4} S_{m_{i}}\left(1-\theta_{i}\right) e^{\lambda_{i} L_{1}}=0, \\
\sum_{i=1}^{4} S_{m_{i}}\left(1-\theta_{i}\right)=0, \\
\sum_{i=1}^{4} S_{m_{i}} e^{\lambda_{i} L_{1}}-S_{m_{5}} e^{-\lambda_{5} L_{2}}-S_{m_{6}} e^{-\lambda_{6} L_{2}}=0, \\
\sum_{i=1}^{4} \lambda_{i} e^{\lambda_{i} L_{1}} S_{m_{i}}\left(A_{1} D_{1}+A_{w} D_{w} \theta_{i}\right)-A_{2} D_{2}\left(S_{m_{5}} \lambda_{5} e^{-\lambda_{5} L_{2}}+S_{m_{6}} \lambda_{6} e^{-\lambda_{6} L_{2}}\right)=0 .
\end{array}\right.
$$

The downstream boundary conditions for $m=n-1$ are

$$
\left\{\begin{array}{l}
\sum_{i=1}^{4} \lambda_{i} S_{(n-1)_{i}}\left(A_{1} D_{1}+A_{w} D_{w} \theta_{i}\right)-A_{2} D_{2} \lambda_{5} S_{(n-2)_{5}}-A_{2} D_{2} \lambda_{6} S_{(n-2)_{6}}=0, \\
\sum_{i=1}^{4} S_{(n-1)_{i}}-S_{(n-2)_{5}}-S_{(n-2)_{6}}=0, \\
\sum_{i=1}^{4} S_{(n-1)_{i}}\left(1-\theta_{i}\right) e^{\lambda_{i} L_{1}}=0, \\
\sum_{i=1}^{4} S_{(n-1)_{i}}\left(1-\theta_{i}\right)=0, \\
\sum_{i=1}^{4} S_{(n-1)_{i}} e^{\lambda_{i} L_{1}}-S_{(n-1)_{5}} e^{-\lambda_{5} L_{2}}-S_{(n-1)_{6}} e^{-\lambda_{6} L_{2}}=0, \\
\sum_{i=1}^{4} \lambda_{i} e^{\lambda_{i} L_{1}} S_{(n-1)_{i}}\left(A_{1} D_{1}+A_{w} D_{w} \theta_{i}\right)-A_{2} D_{2}\left(S_{(n-1)_{5}} \lambda_{5} e^{-\lambda_{5} L_{2}}+S_{(n-1)_{6}} \lambda_{6} e^{-\lambda_{6} L_{2}}\right), \\
\lambda_{5} S_{(n-1)_{5}}+\lambda_{6} S_{(n-1)_{6}}=0 .
\end{array}\right.
$$

We denote

$$
\xi^{T}=\left[S_{0_{1}}, S_{0_{2}}, S_{0_{3}}, S_{0_{4}}, S_{0_{5}}, S_{0_{6}}, S_{1_{1}}, \cdots, S_{(n-1)_{1}}, S_{(n-1)_{2}}, S_{(n-1)_{3}}, S_{(n-1)_{4}}, S_{(n-1)_{5}}, S_{(n-1)_{6}}\right] .
$$

Then all the above matching conditions can be written as $\overline{\mathbf{M}} \xi=0$ with

$$
\overline{\mathbf{M}}=\left[\begin{array}{cccccc}
N_{1} & 0 & 0 & \cdots & 0 & 0 \\
M_{1} & 0 & 0 & \cdots & 0 & 0 \\
M_{2} & M_{3} & 0 & \cdots & 0 & 0 \\
0 & M_{4} & M_{5} & \cdots & 0 & 0 \\
0 & 0 & M_{1} & \cdots & 0 & 0 \\
\vdots & \vdots & \vdots & \ddots & \vdots & \vdots \\
0 & 0 & 0 & \cdots & M_{5} & 0 \\
0 & 0 & 0 & \cdots & M_{1} & 0 \\
0 & 0 & 0 & \cdots & M_{2} & M_{3} \\
0 & 0 & 0 & \cdots & 0 & N_{2}
\end{array}\right]
$$

where $\gamma_{i}=\left(A_{1} D_{1}+A_{w} D_{w} \theta_{i}\right)$. The entries of this block matrix are

$$
\begin{gathered}
N_{1}=\left(\begin{array}{cccc}
\gamma_{1} \lambda_{1}-v_{1}-v_{w} \theta_{1} & \gamma_{2} \lambda_{2}-v_{1}-v_{w} \theta_{2} & \gamma_{3} \lambda_{3}-v_{1}-v_{w} \theta_{3} & \gamma_{4} \lambda_{4}-v_{1}-v_{w} \theta_{4}
\end{array}\right), \\
M_{1}=\left(\begin{array}{cccc}
\left(1-\theta_{1}\right) e^{\lambda_{1} L_{1}} & \left(1-\theta_{2}\right) e^{\lambda_{2} L_{1}} & \left(1-\theta_{3}\right) e^{\lambda_{3} L_{1}} & \left(1-\theta_{4}\right) e^{\lambda_{4} L_{1}} \\
1-\theta_{1} & 1-\theta_{2} & 1-\theta_{3} & 1-\theta_{4}
\end{array}\right),
\end{gathered}
$$




$$
\begin{aligned}
& M_{2}=\left(\begin{array}{cccc}
e^{\lambda_{1} L_{1}} & e^{\lambda_{2} L_{1}} & e^{\lambda_{3} L_{1}} & e^{\lambda_{4} L_{1}} \\
\lambda_{1} \gamma_{1} e^{\lambda_{1} L_{1}} & \lambda_{2} \gamma_{2} e^{\lambda_{2} L_{1}} & \lambda_{3} \gamma_{3} e^{\lambda_{3} L_{1}} & \lambda_{4} \gamma_{4} e^{\lambda_{4} L_{1}}
\end{array}\right), \\
& M_{3}=\left(\begin{array}{cc}
-A_{2} D_{2} \lambda_{5} e^{-\lambda_{5} L_{2}} & -A_{2} D_{2} \lambda_{6} e^{-\lambda_{6} L_{2}} \\
-e^{-\lambda_{5} L_{2}} & -e^{-\lambda_{6} L_{2}}
\end{array}\right) \\
& M_{4}=\left(\begin{array}{cc}
-A_{2} D_{2} \lambda_{5} & -A_{2} D_{2} \lambda_{6} \\
-1 & -1
\end{array}\right), \\
& M_{5}=\left(\begin{array}{cccc}
\lambda_{1} \gamma_{1} & \lambda_{2} \gamma_{2} & \lambda_{3} \gamma_{3} & \lambda_{4} \gamma_{4} \\
1 & 1 & 1 & 1
\end{array}\right) \text {, } \\
& N_{2}=\left(\begin{array}{ll}
\lambda_{5} & \lambda_{6}
\end{array}\right) \text {. }
\end{aligned}
$$

Theorem 3.2 The persistence boundary for the model (6) on the bounded domain $[0, n L]$ with no-flux upstream and free-flow downstream conditions and interface conditions (7) and (9) is given by $\operatorname{det} \overline{\mathbf{M}}=0$ with $\overline{\mathbf{M}}$ as in (31).

If there is a positive solution of $\overline{\mathbf{M}} \xi=0$, we may assume $S_{(n-1)_{6}}=1$. Then $S_{(n-1)_{5}}=$ $-\lambda_{6} / \lambda_{5}$. Successively, we compute

$$
\left(\begin{array}{c}
M_{1} \\
M_{2}
\end{array}\right)\left(\begin{array}{c}
S_{(n-1)_{1}} \\
S_{(n-1)_{2}} \\
S_{(n-1)_{3}} \\
S_{(n-1)_{4}}
\end{array}\right)=-\left(\begin{array}{c}
0 \\
M_{3}
\end{array}\right)\left(\begin{array}{c}
S_{(n-1)_{5}} \\
S_{(n-1)_{6}}
\end{array}\right) \text { and } M_{4}\left(\begin{array}{c}
S_{(n-2)_{5}} \\
S_{(n-2)_{6}}
\end{array}\right)=-M_{5}\left(\begin{array}{c}
S_{(n-1)_{1}} \\
S_{(n-1)_{2}} \\
S_{(n-1)_{3}} \\
S_{(n-1)_{4}}
\end{array}\right) \text {. }
$$

Following this rule, we can obtain all $S_{m_{i}}$. Applying the first row in $\overline{\mathbf{M}}$, we can then obtain the persistence boundary for the population in a bounded domain.

\section{Minimal wave speeds of traveling periodic waves}

We now turn to studying the speed with which a locally introduced population may spread in a river and how this speed depends on the presence and properties of point bars. Numerous previous studies showed that this speed is closely related to the ability of a population to persist in a stretch of a river as follows. In the absence of any drift or movement bias, population spread in a habitat is independent of direction. In a river, the unidirectional water flow yields faster spread in the downstream than the upstream direction. In fact, the individual movement bias induced by river flow is too strong, a locally introduced population will not be able to spread upstream and instead be washed downstream. This is the case exactly if the population is unable to persist in a river of any finite length [Pachepsky et al., 2005, Lutscher et al., 2005, 2010]. The point bars with favorable conditions in meandering rivers are then expected to help individuals move to the upstream against the water flow and persist, unless the flow speeds in the main channel become too high. 
Mathematically, the speed at which a locally introduced population spreads is measured by the asymptotic spreading speed [Weinberger, 1982, 2002]. In practice, this quantity can only be calculated explicitly when the linear conjecture holds, i.e. when the speed can be obtained as the minimal wave speed of the linearized (at zero) equations. Since the growth function in our model does not have an Allee effect, we may assume that the 'linear conjecture' holds. Therefore, we use the linearized equations and make the ansatz of a traveling periodic wave (TPW) [Shigesada et al., 1986, Lutscher et al., 2006]. From it, we will derive the dispersion relation between the speed of the TPW and its decay rate at the leading edge. We will illustrate how the upstream and downstream spread rates depend on parameter values, and particularly, examine how the existence of a point bar in a meandering river affects upstream spread rates and thereby persistence.

\subsection{Derivation of the dispersion relation}

As indicated, we consider the linearized system

$$
\left\{\begin{array}{l}
u_{t}=D_{1} u_{x x}-v_{1} u_{x}+\alpha_{u}(w-u)+r_{1} u, \quad x \in\left(k L, k L+L_{1}\right), \\
w_{t}=D_{w} w_{x x}-v_{w} w_{x}+\alpha_{w}(u-w)+r_{w} w, \quad x \in\left(k L, k L+L_{1}\right), \\
u_{t}=D_{2} u_{x x}-v_{2} u_{x}+r_{2} u, \quad x \in\left(k L+L_{1},(k+1) L\right),
\end{array}\right.
$$

under the interface conditions (7) and (9). We will assume a solution of (32) in the form of a TPW with speed $c$ and shape parameter $s$, given by $u(x, t)=\phi_{u}(x-c t) \psi_{u}(x)$ and $w(x, t)=$ $\phi_{w}(x-c t) \psi_{w}(x)$, where $\psi_{u}$ and $\psi_{w}$ are $L$-periodic functions. Substituting this ansatz into equations (32), we obtain

$$
\left\{\begin{array}{l}
-c \phi_{u}^{\prime} \psi_{u}=\left(D_{1}\left(\phi_{u}^{\prime} \psi_{u}+\phi_{u} \psi_{u}^{\prime}\right)\right)^{\prime}-\left(v_{1} \phi_{u} \psi_{u}\right)^{\prime}+\left(r_{1}-\alpha_{u}\right) \phi_{u} \psi_{u}+\alpha_{u} \phi_{w} \psi_{w} \\
-c \phi_{w}^{\prime} \psi_{w}=\left(D_{w}\left(\phi_{w}^{\prime} \psi_{w}+\phi_{w} \psi_{w}^{\prime}\right)\right)^{\prime}-\left(v_{w} \phi_{w} \psi_{w}\right)^{\prime}+\left(r_{w}-\alpha_{w}\right) \phi_{w} \psi_{w}+\alpha_{w} \phi_{u} \psi_{u} \\
-c \phi_{u}^{\prime} \psi_{u}=\left(D_{2}\left(\phi_{u}^{\prime} \psi_{u}+\phi_{u} \psi_{u}^{\prime}\right)\right)^{\prime}-\left(v_{2} \phi_{u} \psi_{u}\right)^{\prime}+r_{2} \phi_{u} \psi_{u}
\end{array}\right.
$$

Dividing the above equations by $\phi_{u} \psi_{u}$ or $\phi_{w} \psi_{w}$, respectively, yields

$$
\left\{\begin{array}{l}
-c \frac{\phi_{u}^{\prime}}{\phi_{u}}-D_{1} \frac{\phi_{u}^{\prime \prime}}{\phi_{u}}-2 D_{1} \frac{\phi_{u}^{\prime}}{\phi_{u}} \frac{\psi_{u}^{\prime}}{\psi_{u}}+v_{1} \frac{\phi_{u}^{\prime}}{\phi_{u}}-\alpha_{u} \frac{\phi_{w}}{\phi_{u}} \frac{\psi_{w}}{\psi_{u}}=D_{1} \frac{\psi_{u}^{\prime \prime}}{\psi_{u}}-v_{1} \frac{\psi_{u}^{\prime}}{\psi_{u}}+\left(r_{1}-\alpha_{u}\right), \\
-c \frac{\phi_{w}^{\prime}}{\phi_{w}}-D_{w} \frac{\phi_{w}^{\prime \prime}}{\phi_{w}}-2 D_{w} \frac{\phi_{w}^{\prime}}{\phi_{w}} \frac{\psi_{w}^{\prime}}{\psi_{w}}+v_{w} \frac{\phi_{w}^{\prime}}{\phi_{w}}-\alpha_{w} \frac{\phi_{u}}{\phi_{w}} \frac{\psi_{u}}{\psi_{w}}=D_{w} \frac{\psi_{w}^{\prime \prime}}{\psi_{w}}-v_{w} \frac{\psi_{w}^{\prime}}{\psi_{w}}+\left(r_{w}-\alpha_{w}\right), \\
-c \frac{\phi_{u}^{\prime}}{\phi_{u}}-D_{2} \frac{\phi_{u}^{\prime \prime}}{\phi_{u}}-2 D_{2} \frac{\phi_{u}^{u}}{\phi_{u}} \frac{\psi_{u}^{u}}{\psi_{u}}+v_{2} \frac{\phi_{u}^{\prime}}{\phi_{u}}=D_{2} \frac{\psi_{u}^{\prime \prime}}{\psi_{u}}-v_{2} \frac{\psi_{u}^{\prime}}{\psi_{u}}+r_{2}
\end{array}\right.
$$

The third equation in (34) can only be true for independent functions $\phi_{u}(z)$ and $\psi_{u}(x)$ if $\frac{\phi_{u}^{\prime}}{\phi_{u}}$ or $\frac{\psi_{u}^{\prime}}{\psi_{u}}$ is constant. If $\frac{\psi_{u}^{\prime}}{\psi_{u}}$ is a constant then $\psi_{u}$ is an exponential function, which contradicts the requirement that $\psi_{u}$ be periodic. Therefore, we can assume $\frac{\phi_{u}^{\prime}}{\phi_{u}} \equiv$ const $:=-s$. The first equation in (34) then implies that $\phi_{w} / \phi_{u}$ is a constant. Without loss of generality, we assume $\phi_{w}=\phi_{u}$ so that

$$
\phi_{u}(z)=\phi_{w}(z)=e^{-s z}
$$


Hence, the wave front decays exponentially with rate $s$ and the wave shape is determined by the periodic functions $\psi_{u}$ and $\psi_{w}$. The condition that a nontrivial solution for $\psi_{u}$ and $\psi_{w}$ exists then results in the dispersion relation between speed $c$ and decay rate $s$. Minimizing $c(s)$ will give us the minimal wave speed, and - by the above reasoning - the spreading speed.

The equations for $\psi_{u}, \psi_{w}$ on any period are given by

$$
\left\{\begin{array}{l}
D_{1} \psi_{u}^{\prime \prime}-\left(2 D_{1} s+v_{1}\right) \psi_{u}^{\prime}+\psi_{u}\left(-c s+D_{1} s^{2}+v_{1} s+r_{1}-\alpha_{u}\right)+\alpha_{u} \psi_{w}=0, \quad x \in\left(0, L_{1}\right), \\
D_{w} \psi_{w}^{\prime \prime}-\left(2 D_{w} s+v_{w}\right) \psi_{w}^{\prime}+\psi_{w}\left(-c s+D_{w} s^{2}+v_{w} s+r_{w}-\alpha_{w}\right)+\alpha_{w} \psi_{u}=0, \quad x \in\left(0, L_{1}\right), \\
D_{2} \psi_{u}^{\prime \prime}-\left(2 D_{2} s+v_{2}\right) \psi_{u}^{\prime}+\psi_{u}\left(-c s+D_{2} s^{2}+v_{2} s+r_{2}\right)=0, \quad x \in\left(L_{1}, L\right) .
\end{array}\right.
$$

We will state the interface and boundary conditions below. To simplify notation, we define the following expressions:

$$
\begin{aligned}
& K_{1}=2 D_{1} s+v_{1}, \\
& K_{2}=2 D_{2} s+v_{2}, \\
& K_{w}=2 D_{w} s+v_{w}, \\
& H_{1}=-c s+D_{1} s^{2}+v_{1} s+r_{1}-\alpha_{u}, \\
& H_{2}=-c s+D_{2} s^{2}+v_{2} s+r_{2}, \\
& H_{w}=-c s+D_{w} s^{2}+v_{w} s+r_{w}-\alpha_{w} .
\end{aligned}
$$

Then we solve the first equation in (35) for $\psi_{w}$ and differentiate $\psi_{w}$ to obtain

$$
\begin{aligned}
& \psi_{w}=-\frac{1}{\alpha_{u}}\left[D_{1} \psi_{u}^{\prime \prime}-K_{1} \psi_{u}^{\prime}+H_{1} \psi_{u}\right], \\
& \psi_{w}^{\prime}=-\frac{1}{\alpha_{u}}\left[D_{1} \psi_{u}^{\prime \prime \prime}-K_{1} \psi_{u}^{\prime \prime}+H_{1} \psi_{u}^{\prime}\right], \\
& \psi_{w}^{\prime \prime}=-\frac{1}{\alpha_{u}}\left[D_{1} \psi_{u}^{(4)}-K_{1} \psi_{u}^{\prime \prime \prime}+H_{1} \psi_{u}^{\prime \prime}\right] .
\end{aligned}
$$

Substituting the above expressions into the equation for $\psi_{w}$ in (35) leads to the fourth-order equation

$D_{1} D_{w} \psi_{u}^{(4)}-\left(D_{w} K_{1}+D_{1} K_{w}\right) \psi_{u}^{\prime \prime \prime}+\left(D_{w} H_{1}+H_{w} D_{1}+K_{1} K_{w}\right) \psi_{u}^{\prime \prime}-\left(K_{1} H_{w}+K_{w} H_{1}\right) \psi_{u}^{\prime}+\left(H_{1} H_{w}-\alpha_{u} \alpha_{w}\right) \psi_{u}=0$

The general solution of this equation can be expressed as

$$
\psi_{u}(x)=U_{1} e^{\mu_{1} x}+U_{2} e^{\mu_{2} x}+U_{3} e^{\mu_{3} x}+U_{4} e^{\mu_{4} x}, \quad x \in\left(0, L_{1}\right),
$$

where $U_{i}$ are constants, and $\mu_{i}$ are the roots of the characteristic equation of (38), that is, $\mu_{i}$ satisfy

$D_{1} D_{w} \mu_{i}^{4}-\left(D_{w} K_{1}+D_{1} K_{w}\right) \mu_{i}^{3}+\left(D_{w} H_{1}+H_{w} D_{1}+K_{1} K_{w}\right) \mu_{i}^{2}-\left(K_{1} H_{w}+K_{w} H_{1}\right) \mu_{i}+\left(H_{1} H_{w}-\alpha_{u} \alpha_{w}\right)=0$,

provided that (40) does not have multiple solutions. Accordingly, function $\psi_{w}$ is given by

$$
\psi_{w}(x)=W_{1} e^{\mu_{1} x}+W_{2} e^{\mu_{2} x}+W_{3} e^{\mu_{3} x}+W_{4} e^{\mu_{4} x}, \quad x \in\left(0, L_{1}\right),
$$


with

$$
W_{i}=\delta_{i} U_{i}, \quad \delta_{i}=-\frac{D_{1} \mu_{i}^{2}-K_{1} \mu_{i}+H_{1}}{\alpha_{u}}, \quad i=1,2,3,4 .
$$

We can express $\psi_{u}$ on $\left(L_{1}, L\right)$ as

$$
\psi_{u}(x)=Z_{1} e^{-\lambda_{1}(L-x)}+Z_{2} e^{-\lambda_{2}(L-x)}, \quad \lambda_{1,2}=\frac{K_{2} \pm \sqrt{K_{2}^{2}-4 D_{2} H_{2}}}{2 D_{2}} .
$$

Using the matching conditions (7), (9) and (12), we obtain a system of equations for coefficients $U_{i}$ and $Z_{i}$. The first two equations come from the differential equations, the next four from the matching conditions, as indicated in brackets:

$$
\begin{array}{cc}
\sum_{i=1}^{4}\left(A_{1} D_{1}+A_{w} D_{w} \delta_{i}\right) U_{i} e^{\mu_{i} L_{1}}\left(-s+\mu_{i}\right) & -\sum_{i=1}^{2} A_{2} D_{2} Z_{i} e^{-\lambda_{i} L_{2}}\left(-s+\lambda_{i}\right)=0 \\
\sum_{i=1}^{4}\left(A_{1} D_{1}+A_{w} D_{w} \delta_{i}\right) U_{i}\left(-s+\mu_{i}\right)-\sum_{i=1}^{2} A_{2} D_{2} Z_{i} e^{-s L}\left(-s+\lambda_{i}\right)=0 & \left(\text { for } \psi_{u}\left(0^{+}\right)=\psi_{u}\left(L^{-}\right)\right), \\
\sum_{i=1}^{4} U_{i}-Z_{1}-Z_{2}=0, & \left(\text { for } \psi_{u}\left(L_{1}^{-}\right)=\psi_{u}\left(L_{1}^{+}\right)\right), \\
\sum_{i=1}^{4} U_{i} e^{\mu_{i} L_{1}}-\sum_{i=1}^{2} Z_{i} e^{-\lambda_{i} L_{2}}=0, & \left(\text { for } \psi_{u}\left(0^{+}\right)=\psi_{w}\left(0^{+}\right)\right) \\
\sum_{i=1}^{4} U_{i}\left(1-\delta_{i}\right)=0 . & \left(\text { for } \psi_{u}\left(L_{1}^{-}\right)=\psi_{w}\left(L_{1}^{-}\right)\right) . \\
\sum_{i=1}^{4} U_{i}\left(1-\delta_{i}\right) e^{\mu_{i} L_{1}}=0, &
\end{array}
$$

By setting $\xi^{T}=\left[U_{1}, U_{2}, U_{3}, U_{4}, Z_{1}, Z_{2}\right]$ we can write the linear system in (43-48) as $\mathcal{A} \xi=0$ with

$$
\mathcal{A}=\left(\begin{array}{cccccc}
e^{\mu_{1} L_{1}} \sigma_{1} \eta_{1} & e^{\mu_{2} L_{1}} \sigma_{2} \eta_{2} & e^{\mu_{3} L_{1}} \sigma_{3} \eta_{3} & e^{\mu_{4} L_{1}} \sigma_{4} \eta_{4} & -\varsigma_{1} e^{-\lambda_{1} L_{2}} & -\varsigma_{2} e^{-\lambda_{2} L_{2}} \\
\sigma_{1} \eta_{1} & \sigma_{2} \eta_{2} & \sigma_{3} \eta_{3} & \sigma_{4} \eta_{4} & -\varsigma_{1} e^{-s L} & -\varsigma_{2} e^{-s L} \\
1 & 1 & 1 & 1 & -1 & -1 \\
e^{\mu_{1} L_{1}} & e^{\mu_{2} L_{1}} & e^{\mu_{3} L_{1}} & e^{\mu_{4} L_{1}} & -e^{-\lambda_{1} L_{2}} & -e^{-\lambda_{2} L_{2}} \\
e^{\mu_{1} L_{1}}\left(1-\delta_{1}\right) & e^{\mu_{2} L_{1}}\left(1-\delta_{2}\right) & e^{\mu_{3} L_{1}}\left(1-\delta_{3}\right) & e^{\mu_{4} L_{1}}\left(1-\delta_{4}\right) & 0 & 0 \\
1-\delta_{1} & 1-\delta_{2} & 1-\delta_{3} & 1-\delta_{4} & 0 & 0
\end{array}\right)
$$

and $\eta_{i}=A_{1} D_{1}+A_{w} D_{w} \delta_{i}, \sigma_{i}=-s+\mu_{i}, \varsigma_{i}=A_{2} D_{2}\left(-s+\lambda_{i}\right)$.

We obtain a non-trivial solution for $\xi$ only if the determinant for $\mathcal{A}$ is equal to zero. This condition defines the dispersion relation between $c$ and $s$.

Theorem 4.1 The minimal wave speeds of spatially periodic traveling waves of (6) with the interface conditions (7) and (9) in the upstream $\left(c^{-}\right)$and downstream $\left(c^{+}\right)$directions are defined 
as

$$
c^{-}=\max _{s<0}\{c(s)\} \quad \text { and } \quad c^{+}=\min _{s>0}\{c(s)\},
$$

where $c(s)$ is defined by the relation $\operatorname{det} \mathcal{A}=0$.

Remark 4.1 According to our choice of positive flow speeds $v_{1}, v_{2}$ and $v_{w}$, water flow is to the right on the real line. The speeds defined in the preceding theorem are signed speeds in the sense that $c^{+}$or $c^{-}>0$ indicates that the profile moves to the right (downstream) and $c^{+}$or $c^{-}<0$ stands for movement to the left (upstream). In particular, the population can invade upstream if $c^{-}<0$.

\subsection{Numerical simulations for minimal wave speeds}

To illustrate the theoretical results about minimal wave speeds, we conduct a numerical sensitivity analysis similar to what we did in Section 3. Specifically, we numerically calculate the minimal wave speeds of a TPW in the upstream $\left(c^{-}\right)$and downstream $\left(c^{+}\right)$directions from (49). Under the same scenarios as in Section 3, we study how the speeds change when each of the parameters increases from its baseline value by $10 \%$ or $50 \%$. We say that a parameter has a positive (negative) effect if the (absolute) value of the speed increases (decreases).

\subsubsection{Unfavorable main channel}

We first consider the CMC scenario with an unfavorable, constant main channel and a favorable point bar. For the baseline parameters as in the second row of Table 3, the minimal wave speeds in the upstream and downstream directions are $c^{-}=-0.597$ and $c^{+}=1.723$, respectively, which implies that traveling waves spread upstream (downstream) at a speed of at least 0.597 (1.723). In the table, we only record the absolute value of $c^{-}$since $c^{-}$is negative for all parameter settings explored here. As shown in Table 3, parameters $D_{w}, r_{1}, L_{1}, D_{2}, D_{1}, A_{w}$, and $r_{w}$ have a positive effect on $c^{-}$and help the population spread upstream more quickly, while parameters $\alpha_{u}, A_{1}, v_{1}, v_{w}$, and $L_{2}$ have negative effect on $c^{-}$and slow down population spread upstream.

The parameters that have a positive effect on the upstream spread rate are those that contribute to population growth directly (increased growth rate, $r_{w}$, or decreased loss rate, $r_{1}$ ) or indirectly (larger point bar, $L_{1}, A_{w}$ ) or all the diffusion coefficients that contribute to movement. Negative effects on upstream spread result from larger sink areas $\left(A_{1}, L_{2}\right)$ or shorter residence time in point bars $\left(\alpha_{u}\right)$, or faster downstream flow $\left(v_{1}, v_{w}\right)$. Downstream spread, on the other hand, slows down only due to growth-related parameters $\left(\alpha_{u}, A_{1}, L_{2}\right)$ but speeds up with all the other parameters. Obviously, flow speed accelerates downstream spread.

With regards to persistence conditions, we saw above that an increase in the area of the point bar (roughly corresponding to an increase in sinuosity) has two antagonistic effects: increased growth rate and increased flow velocity in one part of the main channel. For upstream spread, these two effects are still antagonistic. For the parameters chosen here, the positive effect of 
increased growth is stronger. For downstream spread, the two effects are not antagonistic but contribute both to increase the downstream speed.

\begin{tabular}{|c|c|c|c|c|c|c|c|c|c|c|c|c|}
\hline parameter $(p)$ & $\alpha_{u}$ & $A_{1}$ & $v_{1}$ & $D_{w}$ & $r_{1}$ & $v_{w}$ & $L_{1}$ & $D_{2}$ & $D_{1}$ & $A_{w}$ & $r_{w}$ & $L_{2}$ \\
\hline \hline baseline value & 0.4 & 1 & 1 & 1 & -0.1 & 0.1 & 1 & 1 & 1 & 1 & 1 & 1 \\
\hline \hline$\left|c^{-}\right|$if $p \uparrow 10 \%$ & 0.589 & 0.577 & 0.571 & 0.605 & 0.599 & 0.593 & 0.609 & 0.625 & 0.603 & 0.617 & 0.653 & 0.587 \\
\hline$\left|c^{-}\right|$if $p \uparrow 50 \%$ & 0.561 & 0.503 & 0.483 & 0.629 & 0.611 & 0.581 & 0.659 & 0.723 & 0.623 & 0.669 & 0.861 & 0.559 \\
\hline effect on $c^{-}$ & - & - & - & + & + & - & + & + & + & + & + & - \\
\hline \hline$c^{+}$if $p \uparrow 10 \%$ & 1.721 & 1.701 & 1.787 & 1.729 & 1.733 & 1.727 & 1.733 & 1.749 & 1.729 & 1.739 & 1.781 & 1.711 \\
\hline$c^{+}$if $p \uparrow 50 \%$ & 1.721 & 1.623 & 2.053 & 1.761 & 1.779 & 1.755 & 1.771 & 1.847 & 1.755 & 1.783 & 1.999 & 1.673 \\
\hline effect on $c^{+}$ & - & - & + & + & + & + & + & + & + & + & + & - \\
\hline
\end{tabular}

Table 3: Illustration of minimal traveling periodic wave speeds according to (49). We present the sensitivity of $c^{ \pm}$to changes in model parameters in the case of an unfavorable, constant crosssection main channel (CMC). Model parameters are in the first row; their baseline values in the second. These baseline values give a minimal upstream wave speed $c^{-}=-0.597$ and a minimal downstream wave speed $c^{+}=1.723$. The third (fourth) row gives $\left|c^{-}\right|$attained when only the corresponding parameter is increased by $10 \%$ (50\%), while the fifth and sixth rows describe similar results for $c^{+}$. All values of $c^{-}$are negative so that the population can spread upstream. Other parameter values are $r_{2}=r_{1}, A_{2}=A_{1}, \alpha_{w}=\alpha_{u} A_{1} / A_{w}, v_{2}=\left(A_{1} v_{1}+A_{w} v_{w}\right) / A_{2}$.

\subsubsection{Alternating main channel}

In the CCS scenario with alternating unfavorable and favorable patches in the main channel and a constant total area, the minimal wave speeds in the upstream and downstream directions are $c^{-}=-0.737$ and $c^{+}=1.293$, respectively, for the chosen baseline parameters as in the second row of Table 4. Again, we record the absolute value of $c^{-}$since $c^{-}$is always negative in this simulation. In Table 4, the sign of the relationship between the speeds and parameters are the same as in Table 3, which implies that in the scenarios of CMC and CCS, parameters have similar effects on population spread in a meandering river.

\section{Discussion}

Meandering rivers represent a common and typical type of free-flowing rivers. The main channel and the point bars in such rivers provide different environments for species living there since hydrological and biological conditions may differ significantly between in these regions. With increased damming and channelization, the differences between these environments might diminish. Engineering efforts around the world recognize the importance of creating and maintaining different habitat types in river restoration. Our work offers a first approach to assess the importance of meandering structures in an analytic framework.

The study of process-based mathematical models for population dynamics in rivers began with the work by Speirs and Gurney [2001] and has since progressed significantly; see references in the introduction. It has become clear that the simplest reaction-advection-diffusion type models cannot correctly predict the realistic range of parameters that guarantee popula- 


\begin{tabular}{|c|c|c|c|c|c|c|c|c|c|c|c|c|}
\hline parameter $(p)$ & $\alpha_{u}$ & $A_{2}$ & $v_{2}$ & $D_{w}$ & $r_{1}$ & $L_{1}$ & $D_{2}$ & $D_{1}$ & $A_{w}$ & $r_{w}$ & $L_{2}$ & $r_{2}$ \\
\hline \hline value of $p$ & 0.5 & 2 & 1 & 1 & 1 & 1 & 1 & 1 & 1 & 2 & 5 & -2 \\
\hline \hline$c^{-}$if $p \uparrow 10 \%$ & 0.731 & 0.717 & 0.717 & 0.741 & 0.741 & 0.745 & 0.777 & 0.739 & 0.757 & 0.801 & 0.731 & 0.751 \\
\hline$c^{-}$if $p \uparrow 50 \%$ & 0.709 & 0.645 & 0.643 & 0.751 & 0.765 & 0.777 & 0.917 & 0.747 & 0.815 & 1.047 & 0.717 & 0.821 \\
\hline effect on $c^{-}$ & - & - & - & + & + & + & + & + & + & + & - & + \\
\hline \hline$c^{+}$if $p \uparrow 10 \%$ & 1.285 & 1.267 & 1.327 & 1.295 & 1.303 & 1.309 & 1.335 & 1.295 & 1.323 & 1.385 & 1.279 & 1.341 \\
\hline$c^{+}$if $p \uparrow 50 \%$ & 1.257 & 1.161 & 1.469 & 1.303 & 1.415 & 1.385 & 1.483 & 1.299 & 1.405 & 1.719 & 1.241 & 1.581 \\
\hline effect on $c^{+}$ & - & - & + & + & + & + & + & + & + & + & - & + \\
\hline
\end{tabular}

Table 4: Illustration of minimal traveling periodic wave speeds according to (49). We present the sensitivity of $c^{ \pm}$to changes in model parameters in the case of an constant cross-section (CCS). Model parameters are in the first row; their baseline values in the second. These baseline values give a minimal upstream wave speed $c^{-}=-0.737$ and a minimal downstream wave speed $c^{+}=1.293$. The third (fourth) row gives $\left|c^{-}\right|$attained when only the corresponding parameter is increased by $10 \%(50 \%)$, while the fifth and sixth rows describe similar results for $c^{+}$. All values of $c^{-}$are negative so that the population can spread upstream. Other parameter values are $A_{1}=A_{2}-A_{w}, \alpha_{w}=\alpha_{u} A_{1} / A_{w}, v_{1}=\left(A_{2} v_{2}-A_{w} v_{w}\right) / A_{1}, v_{w}=0.1$.

tion persistence. In particular, the assumption that flow speeds and population density can be considered constant in a given cross-section seems to simplify the complex structure of a flowingwater environment too much. Adding some structure in flow profile, either continuous [Speirs and Gurney, 2001] or discrete [Pachepsky et al., 2005] offers new mechanisms to promote population persistence. These models still represent a river stretch as a straight channel. Certain aspects of channel geometry were considered by Lutscher et al. [2006] in the form of a poolriffle structure present in many streams. Here, we consider a different, equally salient feature of many streams and rivers, namely the meandering course that creates a main channel, point bars and bluffs. The question of how these features affect population persistence and spread in rivers has not been addressed in an analytical framework before, but first computational models demonstrate that these aspects are important [Jin et al., 2014].

We propose a highly simplified structure of a spatially periodic, meandering river, where we reduce the complex flow patterns to a model of two zones (the main channel and the point bars). This simplification is inspired by the success of a similar division of the vertical flow profile into two compartments [Bencala and Walters, 1983] and the analytical tractability of the resulting models [Pachepsky et al., 2005]. We investigate how this spatial structure affects population persistence (defined as the instability of the trivial solution) and population spread (defined as the minimal wave speed of traveling periodic waves). In both cases, the resulting formulas (for persistence conditions and the minimal wave speeds) are implicit, and we study the sensitivity with respect to single-parameter changes. Comparing the two quantities, persistence and spread, one would expect that parameters that facilitate population growth are beneficial for both, persistence and spread. Parameters that facilitate movement, however, can have different effects on persistence and spread. From the results presented in the four tables, it is clear that increasing growth rates (or decreasing loss rates) make persistence more likely and spread in both directions faster. Flow speed, on the other hand, decreases the likelihood of persistence 
and decreases upstream spread, but increases downstream spread. Diffusion rates have different effects: they increase spread rates in both directions but may or may not increase persistence (expressed as the required area of a point bar of positive population growth). Just as the lateral exchange coefficients $(\alpha)$, diffusion rates influence residence times, and increased residence times in population-dynamic sources help persistence but slow the spread.

If we take the size of a point bar as a proxy for sinuosity of the meandering river, then our results indicate that stronger meanders could benefit a population in terms of both, the ability to persist and spread. While we varied each parameter individually, with some constraints given by the CMC and CCS scenarios, real parameter values are likely to show more correlations. For example, higher sinuosity could also increase the length of the segments. While we found no significant effect of the length of sink patches for population persistence, the spread rates were negatively affected by an increase in $L_{2}$. Future studies could investigate the combined result of these effects with realistic relationships between parameters. Most likely, the outcome will depend on the relative strength of the respective growth rates, and could therefore quite heavily depend on the particular river and/or species. One suggestion forward would be to formulate a model without population growth and study residence times rather than spread rates.

We only conducted our sensitivity analysis of the persistence condition on the unbounded domain. With the implicit formula derived in Section 3.2, one should be able to conduct similar sensitivity analyses on a bounded domain. We expect that they yield qualitatively similar results as in Tables 1 and 2. Moreover, the result that favorable point bars helps population persistence and spread in an infinitely long river is consistent throughout Sections 3 and 4. Therefore, in a bounded (CMC or CCS) meandering river, one should expect to see that the presence of favorable point bars or improvement of favorable conditions in point bars reduces the length (or the number of periods) of the river such that the population can persist (i.e., the critical domain size).

Our model does not include the distinction between water column and benthos that were shown to be important in previous models. Our main reasoning for this simplification was the resulting model complexity would prohibit qualitative insights and complicate the analysis. Future work should include a benthic state and consider the interaction between the different persistence mechanisms that each scenario affords.

Reaction-advection-diffusion equations arise in other applications with uni-directional flow or movement bias. Probably most relevant here is the case of a coastline with long-shore currents, most commonly represented as a straight line [Byers and Pringle, 2006, Pringle et al., 2009]. We speculate that our model could be applied to a scenario of a meandering coastline with more secluded bays and exposed tips. Surprisingly similar models were used to study the distribution, competition and uptake of bacteria in the intestines [Ballyk et al., 1998, Boldin, 2007]. Again, these models idealized the domain as a straight line. Since the intestines are tightly packed and wound, we suggest that the straight-line models have to be modified to represent the winding structure better.

Our model poses many new analytical challenges. We only showed the existence and unique- 
ness of solutions for one set of boundary conditions. This theory should be extended to cover other boundary conditions, and eventually to rigorously prove the existence of a spreading speed and traveling periodic waves, and - if possible - the linear determinacy of the spreading speed. The set-up of our equations is non-standard to capture the geometry of the river, yet simplify the domain to one dimension. It is conceivable that a model for a two-dimensional density on a periodically varying domain has 'nicer' analytical properties. The precise mathematical relationship between such a two- and our one-dimensional model should be explored further. In one or two dimensions, the theory of homogenization, under the assumption that the period of the meanders is small compared to the movement rates of individuals, could yield valuable insights and simplify the analysis of persistence conditions and spread speeds considerably.

\section{Acknowledgements}

YJ gratefully acknowledges an AMS-Simons Travel grant and NSF Grant DMS 1411703. FL is grateful for an NSERC Discovery Grant RGPIN-2016-04795.

\section{Appendix A Existence and uniqueness of solutions}

In this appendix, we prove the existence and uniqueness of model (6) over a bounded periodic domain $[0, n L]$ under the interface conditions (7) and (9), hostile upstream boundary condition and free flow downstream boundary condition, i.e.

$$
u(0, t)=w(0, t)=u_{x}(0, t)=w_{x}(0, t)=0, u_{x}(n L, t)=0 .
$$

Without loss of generality, we provide the proof over a two-period domain $\Omega=[0,2 L]=\Omega_{1} \cup \Omega_{2}$, where $\Omega_{1}=\left[0, L_{1}\right] \cup\left[L, L+L_{1}\right]$ and $\Omega_{2}=\left[L_{1}, L\right] \cup\left[L+L_{1}, 2 L\right]$.

We briefly summarize the key steps of the Galerkin approximation and energy method that we use for the proof. A detailed process can be found in Larios and Pei [2016] and references therein.

Given a PDE system

$$
\frac{d}{d t} u(x, t)=\mathcal{F}(u, \nabla u, \Delta u)
$$

for some smooth function $\mathcal{F}$, we consider a sequence of approximated systems

$$
\frac{d}{d t} P_{\sigma}^{(m)} u(x, t)=\mathcal{F}\left(P_{\sigma}^{(m)} u, \nabla P_{\sigma}^{(m)} u, \Delta P_{\sigma}^{(m)} u\right)
$$

for $m=1,2, \ldots$ after formally applying certain projections $P_{\sigma}^{(m)}$ onto a finite-dimensional subspace of the domain of the differential operator in the equation. Then, we take the inner product of the projected system with the eigenfunctions $\left\{\psi_{i}(x)\right\}_{i=1}^{m}$ of the differential operator, which is positive-definitive, self-adjoint, and compact, and obtain a sequence of ordinary differential 
equations (ODE). Each of the ODE system has a unique solution $\{\alpha(t)\}_{i=1}^{M}$ from which we obtain $P_{\sigma}^{(m)} u(x, t)=\sum_{i=1}^{m} \alpha_{i}(t) \psi_{i}(x)$ for $m=1,2, \ldots$

Then, we obtain the energy estimates for $P_{\sigma}^{(m)} u(x, t)$ in some Banach space $Y$, say, $Y=$ $L^{2}(\Omega)$, as well as the boundedness of those solutions in some space $X$, say, $X=H^{1}(\Omega)$, which is compactly embedded in $Y$. Hence, we can extract a convergent subsequence of the above solutions as a sequence in $Y$, provided that the time derivative of the aforementioned sequence also remains bounded in $Z$, say, the dual space of $X$, and $Y$ is continuously embedded in $Z$. The limit of the above convergent subsequence indeed satisfies the original PDE.

A critical result for the process to work is the Aubin-Lions Compactness Lemma below (see remarks after Lemma 8.2 in Chapter Eight of Constantin and Foias [1988] as well as Lemma 2.1 in Section 2, Chapter 3 of Temam [2001]).

Lemma A.1 Let $T>0$, fix some $p \in(1, \infty)$, and let $\left\{f_{n}(\cdot, t)\right\}_{n=1}^{\infty}$ be a bounded sequence of functions in $L_{t}^{p}(Y ;[0, T])$, where $Y$ is a Banach space. If $\left\{f_{n}\right\}_{n=1}^{\infty}$ is also bounded in $L_{t}^{p}(X ;[0, T])$, where $X$ is compactly imbedded in $Y$, and $\left\{\partial f_{n} / \partial t\right\}_{n=1}^{\infty}$ is uniformly bounded in $L_{t}^{p}(Z ;[0, T])$, where $Y$ is continuously imbedded in $Z$, then $\left\{f_{n}\right\}_{n=1}^{\infty}$ is relatively compact in $L_{t}^{p}(Y ;[0, T])$.

For simplicity, we provide only the key a priori energy estimates under the interface conditions that lead to the boundedness results required in this lemma. We omit the details for constructing solutions via the standard Galerkin approximation; see Constantin and Foias [1988], Temam [2001].

Throughout this section, we use $L^{2}$ and $H^{k}=W^{k, 2}=\left\{u \in L^{2}: D^{\alpha} u \in L^{2}, \forall \alpha\right.$ with $\left.|\alpha| \leq k\right\}$, $k=1,2$, to denote Lebesgue and Sobolev spaces, respectively, while we denote by $C_{0}^{\infty}$ the space of compactly supported smooth functions in space and time.

First, we give the definition of weak solutions to (6).

Definition A.1 Let $T>0$ and suppose that $u_{0}, w_{0} \in L^{2}$ with $u_{0} \geq 0$ and $w_{0} \geq 0$. We say that the pair $(u, w)$ is a weak solution to (6), if $u, w \in L^{\infty}\left(L^{2} ;[0, T]\right) \cap L^{2}\left(H^{1} ;[0, T]\right)$. Furthermore, for test functions $\phi^{(1)} \in C_{0}^{\infty}\left(\left[0, L_{1}\right] \times[0, T]\right), \phi^{(2)} \in C_{0}^{\infty}\left(\left[L, L+L_{1}\right] \times[0, T]\right)$, and $\psi^{(1)} \in C_{0}^{\infty}\left(\left[L_{1}, L\right] \times[0, T]\right), \psi^{(2)} \in C_{0}^{\infty}\left(\left[L+L_{1}, 2 L\right] \times[0, T]\right)$, (6) holds in the weak sense, i.e., 
we have

$$
\begin{aligned}
& \int-\int_{0}^{T} \int_{0}^{L_{1}} u(x, t) \frac{\partial \phi^{(1)}}{\partial t} d x d t-\int_{0}^{T} \int_{L}^{L+L_{1}} u(x, t) \frac{\partial \phi^{(2)}}{\partial t} d x d t \\
& -D_{1} \int_{0}^{T} \int_{0}^{L_{1}} u(x, t) \frac{\partial^{2} \phi^{(1)}}{\partial x^{2}} d x d t-D_{1} \int_{0}^{T} \int_{L}^{L+L_{1}} u(x, t) \frac{\partial^{2} \phi^{(2)}}{\partial x^{2}} d x d t \\
& =v_{1} \int_{0}^{T} \int_{0}^{L_{1}} u(x, t) \frac{\partial \phi^{(1)}}{\partial x} d x d t+v_{1} \int_{0}^{T} \int_{L}^{L+L_{1}} u(x, t) \frac{\partial \phi^{(2)}}{\partial x} d x d t \\
& +\alpha_{u} \int_{0}^{T} \int_{0}^{L_{1}}(w(x, t)-u(x, t)) \phi^{(1)} d x d t+\alpha_{u} \int_{0}^{T} \int_{L}^{L+L_{1}}(w(x, t)-u(x, t)) \phi^{(2)} d x d t \\
& +\int_{0}^{T} \int_{0}^{L_{1}} f_{1}(u(x, t)) u(x, t) \phi^{(1)} d x d t+\int_{0}^{T} \int_{L}^{L+L_{1}} f_{1}(u(x, t)) u(x, t) \phi^{(2)} d x d t, \\
& -\int_{0}^{T} \int_{0}^{L_{1}} w(x, t) \frac{\partial \phi^{(1)}}{d t} d x d t-\int_{0}^{T} \int_{L}^{L+L_{1}} w(x, t) \frac{\partial \phi^{(2)}}{d t} d x d t \\
& -D_{w} \int_{0}^{T} \int_{0}^{L_{1}} w(x, t) \frac{\partial^{2} \phi^{(1)}}{\partial x^{2}} d x d t-D_{w} \int_{0}^{T} \int_{L}^{L+L_{1}} w(x, t) \frac{\partial^{2} \phi^{(2)}}{\partial x^{2}} d x d t \\
& =v_{w} \int_{0}^{T} \int_{0}^{L_{1}} w(x, t) \frac{\partial \phi^{(1)}}{\partial x} d x d t+v_{w} \int_{0}^{T} \int_{L}^{L+L_{1}} w(x, t) \frac{\partial \phi^{(2)}}{\partial x} d x d t \\
& +\alpha_{w} \int_{0}^{T} \int_{0}^{L_{1}}(u(x, t)-w(x, t)) \phi^{(1)} d x d t+\alpha_{w} \int_{0}^{T} \int_{L}^{L+L_{1}}(u(x, t)-w(x, t)) \phi^{(2)} d x d t \\
& +\int_{0}^{T} \int_{0}^{L_{1}} g(w(x, t)) w(x, t) \phi^{(1)} d x d t+\int_{0}^{T} \int_{L}^{L+L_{1}} g(w(x, t)) w(x, t) \phi^{(2)} d x d t, \\
& -\int_{0}^{T} \int_{L_{1}}^{L} u(x, t) \frac{\partial \psi^{(1)}}{d t} d x d t-\int_{0}^{T} \int_{L+L_{1}}^{2 L} u(x, t) \frac{\partial \psi^{(2)}}{d t} d x d t \\
& -D_{2} \int_{0}^{T} \int_{L_{1}}^{L} u(x, t) \frac{\partial^{2} \psi^{(1)}}{\partial x^{2}} d x d t-D_{2} \int_{0}^{T} \int_{L+L_{1}}^{2 L} u(x, t) \frac{\partial^{2} \psi^{(2)}}{\partial x^{2}} d x d t \\
& =v_{2} \int_{0}^{T} \int_{L_{1}}^{L} u(x, t) \frac{\partial \psi^{(1)}}{\partial x} d x d t+v_{2} \int_{0}^{T} \int_{L+L_{1}}^{2 L} u(x, t) \frac{\partial \psi^{(2)}}{\partial x} d x d t
\end{aligned}
$$

In the following proof of the well-posedness of system (6), for the sake of simplicity, we provide only the a priori $L^{2}$-energy and $H^{1}$-enstrophy estimates. We denote $u(\cdot)=u(\cdot, t)$ and similarly for the derivatives of $u$ in space and time.

Proof of Theorem 2.1:

Step 1: $L^{2}$-estimates. Multiply the three equations in (6) by $A_{1} u, A_{w} w$, and $A_{2} u$, respec- 
tively, integrate by parts over $\Omega_{1}$ and $\Omega_{2}$ accordingly, and add, so we obtain

$$
\begin{aligned}
\frac{1}{2} \frac{d}{d t} & \left(A_{1}\|u\|_{L^{2}\left(\Omega_{1}\right)}^{2}+A_{2}\|u\|_{L^{2}\left(\Omega_{2}\right)}^{2}+A_{w}\|w\|_{L^{2}\left(\Omega_{1}\right)}^{2}\right) \\
& +A_{1} D_{1}\left\|u_{x}\right\|_{L^{2}\left(\Omega_{1}\right)}^{2}+A_{2} D_{2}\left\|u_{x}\right\|_{L^{2}\left(\Omega_{2}\right)}^{2}+A_{w} D_{w}\left\|u_{x}\right\|_{L^{2}\left(\Omega_{1}\right)}^{2}+\left(\mathbf{B}_{1}\right) \\
= & \underbrace{-A_{1} v_{1} \int_{\Omega_{1}} u u_{x} d x-A_{2} v_{2} \int_{\Omega_{2}} u u_{x} d x-A_{w} v_{w} \int_{\Omega_{1}} w w_{x} d x}_{I} \\
& \underbrace{+A_{1} \alpha_{1} \int_{\Omega_{1}}(w-u) u d x+A_{w} \alpha_{w} \int_{\Omega_{1}}(u-w) w d x}_{I I} \\
& +\underbrace{+A_{1} \int_{\Omega_{1}} f_{1}(u) u^{2} d x+A_{2} \int_{\Omega_{2}} f_{2}(u) u^{2} d x+A_{w} \int_{\Omega_{1}} g(w) w^{2} d x}_{I I I}
\end{aligned}
$$

where $\left(\mathbf{B}_{\mathbf{1}}\right)$ on the left side of the equation represents the boundary terms, which from integration by parts can be written as

$$
\begin{aligned}
\left(\mathbf{B}_{1}\right)= & A_{1} D_{1} u_{x}(0) u(0)-A_{1} D_{1} u_{x}\left(L_{1}\right) u\left(L_{1}\right)-A_{1} D_{1} u_{x}\left(L+L_{1}\right) u\left(L+L_{1}\right)+A_{1} D_{1} u_{x}(L) u(L) \\
& A_{w} D_{w} w_{x}(0) w(0)-A_{w} D_{w} w_{x}\left(L_{1}\right) w\left(L_{1}\right)-A_{w} D_{w} w_{x}\left(L+L_{1}\right) w\left(L+L_{1}\right)+A_{w} D_{w} w_{x}(L) w(L) \\
& -A_{2} D_{2} u_{x}(L) u(L)+A_{2} D_{2} u_{x}\left(L_{1}\right) u\left(L_{1}\right)-A_{2} D_{2} u_{x}(2 L) u(2 L)+A_{2} D_{2} u_{x}\left(L+L_{1}\right) u\left(L+L_{1}\right) \\
= & A_{1} D_{1} u_{x}(0) u(0)+A_{w} D_{w} w_{x}(0) w(0)-A_{2} D_{2} u_{x}(2 L) u(2 L),
\end{aligned}
$$

by using the interface conditions (9).

In order to estimate the three parts $I, I I$, and $I I I$ on the right side of (52), we first notice that due to the hydrological condition (1) and integration by parts, we have

$$
\begin{aligned}
I= & \frac{1}{2} A_{1} v_{1} u^{2}(0)-\frac{1}{2} A_{1} v_{1} u^{2}\left(L_{1}\right)-\frac{1}{2} A_{1} v_{1} u^{2}\left(L+L_{1}\right)+\frac{1}{2} A_{1} v_{1} u^{2}(L) \\
& \frac{1}{2} A_{w} v_{w} w^{2}(0)-\frac{1}{2} A_{w} v_{w} w^{2}\left(L_{1}\right)-\frac{1}{2} A_{w} v_{w} w^{2}\left(L+L_{1}\right)+\frac{1}{2} A_{w} v_{w} w^{2}(L) \\
& -\frac{1}{2} A_{2} v_{2} u^{2}(L)+\frac{1}{2} A_{2} v_{2} u^{2}\left(L_{1}\right)-\frac{1}{2} A_{2} v_{2} u^{2}(2 L)+\frac{1}{2} A_{2} v_{2} u^{2}\left(L+L_{1}\right) \\
= & \frac{1}{2} A_{1} v_{1} u^{2}(0)+\frac{1}{2} A_{w} v_{w} w^{2}(0)-\frac{1}{2} A_{2} v_{2} u^{2}(2 L) .
\end{aligned}
$$

Therefore, by the boundary conditions (50), we have

$$
\begin{aligned}
I-\left(\mathbf{B}_{\mathbf{1}}\right) & =-\left[A_{1} D_{1} u_{x}(0)+A_{w} D_{w} w_{x}(0)-A_{1} v_{1} u(0)-A_{w} v_{w} w(0)\right] u(0) \\
& -\frac{1}{2} A_{1} v_{1} u^{2}(0)-\frac{1}{2} A_{w} v_{w} w^{2}(0)+\left[A_{2} D_{2} u_{x}(2 L)-\frac{1}{2} A_{2} v_{2} u(2 L)\right] u(2 L) \\
& \leq 0
\end{aligned}
$$


Next, by denoting $A=\max \left\{A_{1}, A_{2}, A_{w}\right\}$ and $\alpha=\max \left\{\alpha_{u}, \alpha_{w}\right\}$, we estimate $I I$ as

$$
I I \leq A \alpha\left(\|u\|_{L^{2}\left(\Omega_{1}\right)}^{2}+\|u\|_{L^{2}\left(\Omega_{2}\right)}^{2}+\|w\|_{L^{2}\left(\Omega_{1}\right)}^{2}\right)
$$

where we used Cauchy-Schwarz inequality. Regarding the remaining integrals, we use the assumption that $f_{1}, f_{2}, g \in L^{\infty}(\Omega)$ and proceed as

$$
I I I \leq A\left(\left\|f_{1}\right\|_{L^{\infty}\left(\Omega_{1}\right)}\|u\|_{L^{2}\left(\Omega_{1}\right)}^{2}+\left\|f_{2}\right\|_{L^{\infty}\left(\Omega_{2}\right)}\|u\|_{L^{2}\left(\Omega_{2}\right)}^{2}+\|g\|_{L^{\infty}\left(\Omega_{1}\right)}\|w\|_{L^{2}\left(\Omega_{1}\right)}^{2}\right)
$$

Thus, by denoting

$$
X^{2}(t)=A_{1}\|u\|_{L^{2}\left(\Omega_{1}\right)}^{2}+A_{2}\|u\|_{L^{2}\left(\Omega_{2}\right)}^{2}+A_{w}\|w\|_{L^{2}\left(\Omega_{1}\right)}^{2},
$$

all the above estimates imply that

$$
\begin{aligned}
& \frac{d}{d t} X^{2}(t)+A_{1} D_{1}\left\|u_{x}\right\|_{L^{2}\left(\Omega_{1}\right)}^{2}+A_{2} D_{2}\left\|u_{x}\right\|_{L^{2}\left(\Omega_{2}\right)}^{2}+A_{w} D_{w}\left\|w_{x}\right\|_{L^{2}\left(\Omega_{1}\right)}^{2} \\
& \leq C X^{2}(t)
\end{aligned}
$$

where for $j=1,2$, the constant $C$ depends on $A_{j}, A_{w}, \alpha_{u}, \alpha_{w}$, and $\left\|f_{j}\right\|_{L^{\infty}(\Omega)},\|g\|_{L^{\infty}(\Omega)}$. Hence, we conclude that $u, w \in L^{\infty}\left(L^{2} ;[0, T]\right) \cap L^{2}\left(H^{1} ;[0, T]\right)$.

Step 2: Uniqueness. Suppose there are two solutions to the system $(6)$, say, $\left(u^{(1)}, w^{(1)}\right)$ and $\left(u^{(2)}, w^{(2)}\right)$, possessing the same initial data and satisfying the identical hostile boundary and interior interface conditions. We denote the difference $\tilde{u}=u^{(1)}-u^{(2)}$ and $\tilde{w}=w^{(1)}-w^{(2)}$ and subtract the systems satisfied by the two solutions. Then, we perform the same energy estimates to the system for $(\tilde{u}, \tilde{w})$ as in Step 1, and obtain

$$
\begin{aligned}
& \frac{d}{d t}\left(A_{1}\|\tilde{u}\|_{L^{2}\left(\Omega_{1}\right)}^{2}+A_{2}\|\tilde{u}\|_{L^{2}\left(\Omega_{2}\right)}^{2}+A_{w}\|\tilde{w}\|_{L^{2}\left(\Omega_{1}\right)}^{2}\right) \\
& \quad+A_{1} D_{1}\left\|\tilde{u}_{x}\right\|_{L^{2}\left(\Omega_{1}\right)}^{2}+A_{2} D_{2}\left\|\tilde{u}_{x}\right\|_{L^{2}\left(\Omega_{2}\right)}^{2}+A_{w} D_{w}\left\|\tilde{w}_{x}\right\|_{L^{2}\left(\Omega_{1}\right)}^{2} \\
& \leq \tilde{C}\left(A_{1}\|\tilde{u}\|_{L^{2}\left(\Omega_{1}\right)}^{2}+A_{2}\|\tilde{u}\|_{L^{2}\left(\Omega_{2}\right)}^{2}+A_{w}\|\tilde{w}\|_{L^{2}\left(\Omega_{1}\right)}^{2}\right)
\end{aligned}
$$

where we assumed that $f_{1}, f_{2}$, and $g$ are Lipschitz continuous. Hence, the uniqueness of the above solution follows from Grönwall's inequality applied to the analogues of (57) with initial value $\tilde{u}_{0}=\tilde{w}_{0}=0$. For simplicity, we skip the details here.

Step 3: Higher regularity ( $H^{1}$-estimates). Assume $u_{0}, w_{0} \in H^{1}$ with $u_{0} \geq 0$ and $w_{0} \geq 0$ and $T>0$. We prove a priori that the solution $(u, w)$ is actually regular on $\Omega \times[0, T]$. Multiply (6) by $-A_{1} D_{1} u_{x x},-A_{w} D_{w} w_{x x}$, and $-A_{2} D_{2} u_{x x}$, respectively, integrate by parts over $\Omega_{1}$ and $\Omega_{2}$ 
accordingly, and add, so that we have

$$
\begin{aligned}
\frac{1}{2} \frac{d}{d t} & \left(A_{1} D_{1}\left\|u_{x}\right\|_{L^{2}\left(\Omega_{1}\right)}^{2}+A_{2} D_{2}\left\|u_{x}\right\|_{L^{2}\left(\Omega_{2}\right)}^{2}+A_{w} D_{w}\left\|w_{x}\right\|_{L^{2}\left(\Omega_{1}\right)}^{2}\right) \\
& +A_{1} D_{1}^{2}\left\|u_{x x}\right\|_{L^{2}\left(\Omega_{1}\right)}^{2}+A_{2} D_{2}^{2}\left\|u_{x x}\right\|_{L^{2}\left(\Omega_{1}\right)}^{2}+A_{w} D_{w}^{2}\left\|w_{x x}\right\|_{L^{2}\left(\Omega_{1}\right)}^{2}+\left(\mathbf{B}_{\mathbf{2}}\right) \\
= & \underbrace{A_{1} D_{1} v_{1} \int_{\Omega_{1}} u_{x} u_{x x} d x+A_{2} D_{2} v_{2} \int_{\Omega_{2}} u_{x} u_{x x} d x+A_{w} D_{w} v_{w} \int_{\Omega_{1}} w_{x} w_{x x} d x}_{I V} \\
& \underbrace{-A_{1} D_{1} \alpha_{u} \int_{\Omega_{1}}(w-u) u_{x x} d x-A_{w} D_{w} \alpha_{w} \int_{\Omega_{1}}(u-w) w_{x x} d x}_{V} \\
& \underbrace{-A_{1} D_{1} \int_{\Omega_{1}} f_{1}(u) u u_{x x} d x-A_{2} D_{2} \int_{\Omega_{2}} f_{2}(u) u u_{x x} d x-A_{w} D_{w} \int_{\Omega_{1}} g(w) w w_{x x} d x}_{V I},
\end{aligned}
$$

where $\left(\mathbf{B}_{\mathbf{2}}\right)$ represents the boundary terms from the left side of $(58)$, which by the interface conditions, add up to 0, i.e.,

$$
\begin{aligned}
\left(\mathbf{B}_{2}\right)= & A_{1} D_{1} u_{t}(0) u_{x}(0)-A_{1} D_{1} u_{t}\left(L_{1}\right) u_{x}\left(L_{1}\right)+A_{1} D_{1} u_{t}(L) u_{x}(L)-A_{1} D_{1} u_{t}\left(L+L_{1}\right) u_{x}\left(L+L_{1}\right) \\
& +A_{w} D_{w} w_{t}(0) w_{x}(0)-A_{w} D_{w} w_{t}\left(L_{1}\right) w_{x}\left(L_{1}\right)+A_{w} D_{w} w_{t}(L) w_{x}(L) \\
& -A_{w} D_{w} w_{t}\left(L+L_{1}\right) w_{x}\left(L+L_{1}\right)+A_{2} D_{2} u_{t}\left(L_{1}\right) u_{x}\left(L_{1}\right)-A_{2} D_{2} u_{t}(L) u_{x}(L) \\
& +A_{2} D_{2} u_{t}\left(L+L_{1}\right) u_{x}\left(L+L_{1}\right)-A_{2} D_{2} u_{t}(2 L) u_{x}(2 L) \\
= & u_{t}(0)\left[A_{1} D_{1} u_{x}(0)+A_{w} D_{w} w_{x}(0)\right]+u_{t}\left(L_{1}\right)\left[-A_{1} D_{1} u_{x}\left(L_{1}\right)-A_{w} D_{w} w_{x}\left(L_{1}\right)+A_{2} D_{2} u_{x}\left(L_{1}\right)\right] \\
& +u_{t}(L)\left[A_{1} D_{1} u_{x}(L)+A_{w} D_{w} w_{x}(L)-A_{2} D_{2} u_{x}(L)\right] \\
& +u_{t}\left(L+L_{1}\right)\left[-A_{1} D_{1} u_{x}\left(L+L_{1}\right)-A_{w} D_{w} w_{x}\left(L+L_{1}\right)+A_{2} D_{2} u_{x}\left(L+L_{1}\right)\right] \\
& -A_{2} D_{2} u_{t}(2 L) u_{x}(2 L) \\
= & u_{t}(0)\left[A_{1} D_{1} u_{x}(0)+A_{w} D_{w} w_{x}(0)\right]-A_{2} D_{2} u_{t}(2 L) u_{x}(2 L) \\
= & 0
\end{aligned}
$$

where we used the condition $u_{t}=w_{t}$ at $x=0, L, L_{1}$, and $L+L_{1}$, and boundary conditions (50). In order to estimate $I V$, we use Cauchy-Schwarz inequality and proceed as

$$
\begin{aligned}
I V \leq & C_{1}\left(\left\|u_{x}\right\|_{L^{2}\left(\Omega_{1}\right)}^{2}+\left\|u_{x}\right\|_{L^{2}\left(\Omega_{2}\right)}^{2}+\left\|w_{x}\right\|_{L^{2}\left(\Omega_{1}\right)}^{2}\right) \\
& +\frac{A_{1} D_{1}^{2}}{16}\left\|u_{x x}\right\|_{L^{2}\left(\Omega_{1}\right)}^{2}+\frac{A_{2} D_{2}^{2}}{16}\left\|u_{x x}\right\|_{L^{2}\left(\Omega_{2}\right)}^{2}+\frac{A_{w} D_{w}^{2}}{16}\left\|w_{x x}\right\|_{L^{2}\left(\Omega_{1}\right)}^{2},
\end{aligned}
$$

where for $j=1,2$, the constant $C_{1}$ depends on $A_{j}, v_{j}$, and $A_{w}, v_{w}$. Next, we integrate by parts 
and bound $V$ as

$$
\begin{aligned}
V= & A_{1} D_{1} v_{1} \int_{\Omega_{1}} u_{x} w_{x} d x-A_{1} D_{1} v_{1} \int_{\Omega_{1}} u_{x}^{2} d x \\
& +A_{w} D_{w} v_{w} \int_{\Omega_{1}} u_{x} w_{x} d x-A_{w} D_{w} v_{w} \int_{\Omega_{1}} w_{x}^{2} d x \\
\leq & C_{2}\left(\left\|u_{x}\right\|_{L^{2}\left(\Omega_{1}\right)}^{2}+\left\|u_{x}\right\|_{L^{2}\left(\Omega_{2}\right)}^{2}+\left\|w_{x}\right\|_{L^{2}\left(\Omega_{1}\right)}^{2}\right),
\end{aligned}
$$

where we used Cauchy-Schwarz inequality. Here the constant $C_{2}$ depends on all parameters including $D_{1}, D_{2}$ and $D_{w}$. Note that the boundary terms from the above integration by parts add up to 0 due to the continuity condition (7). Regarding $V I$, we first apply Hölder's inequality and get

$$
\begin{aligned}
V I \leq & A_{1} D_{1}\left\|f_{1}\right\|_{L^{\infty}\left(\Omega_{1}\right)}\|u\|_{L^{2}\left(\Omega_{1}\right)}\left\|u_{x x}\right\|_{L^{2}\left(\Omega_{1}\right)}+A_{2} D_{2}\left\|f_{2}\right\|_{L^{\infty}\left(\Omega_{2}\right)}\|u\|_{L^{2}\left(\Omega_{2}\right)}\left\|u_{x x}\right\|_{L^{2}\left(\Omega_{2}\right)} \\
& +A_{w} D_{w}\|g\|_{L^{\infty}\left(\Omega_{1}\right)}\|w\|_{L^{2}\left(\Omega_{1}\right)}\left\|w_{x x}\right\|_{L^{2}\left(\Omega_{1}\right)} \\
\leq & C_{3}\left(\left\|u_{x}\right\|_{L^{2}\left(\Omega_{1}\right)}^{2}+\left\|u_{x}\right\|_{L^{2}\left(\Omega_{2}\right)}^{2}+\left\|w_{x}\right\|_{L^{2}\left(\Omega_{1}\right)}^{2}\right) \\
& +\frac{A_{1} D_{1}^{2}}{16}\left\|u_{x x}\right\|_{L^{2}\left(\Omega_{1}\right)}^{2}+\frac{A_{2} D_{2}^{2}}{16}\left\|u_{x x}\right\|_{L^{2}\left(\Omega_{2}\right)}^{2}+\frac{A_{w} D_{w}^{2}}{16}\left\|w_{x x}\right\|_{L^{2}\left(\Omega_{1}\right)}^{2},
\end{aligned}
$$

where we also used Cauchy-Schwarz and Poincaré's inequality in the last step. The constant $C_{3}$ in the above estimates depends on $A_{j}, A_{w}, L, L_{1}$, and $\left\|f_{j}\right\|_{L^{\infty}\left(\Omega_{j}\right)},\|g\|_{L^{\infty}\left(\Omega_{1}\right)}$, for $j=1,2$. Summing up all the above estimates, and denoting

$$
Y^{2}(t)=A_{1} D_{1}\left\|u_{x}\right\|_{L^{2}\left(\Omega_{1}\right)}^{2}+A_{2} D_{2}\left\|u_{x}\right\|_{L^{2}\left(\Omega_{2}\right)}^{2}+A_{w} D_{w}\left\|w_{x}\right\|_{L^{2}\left(\Omega_{1}\right)}^{2},
$$

we get

$$
\frac{d}{d t} Y^{2}(t)+\left(A_{1} D_{1}^{2}\left\|u_{x x}\right\|_{L^{2}\left(\Omega_{1}\right)}^{2}+A_{2} D_{2}^{2}\left\|u_{x x}\right\|_{L^{2}\left(\Omega_{1}\right)}^{2}+A_{w} D_{w}^{2}\left\|w_{x x}\right\|_{L^{2}\left(\Omega_{1}\right)}^{2}\right) \leq C Y^{2}(t),
$$

where $C$ depends on $A_{j}, v_{j}, \alpha_{u}, \alpha_{w}$, and $A_{w}, v_{w}$, for $j=1,2$. Thus, Grönwall's inequality implies that $u, w \in L^{\infty}\left(H^{1} ;[0, T]\right) \cap L^{2}\left(H^{2} ;[0, T]\right)$. Hence, the proof is complete.

Remark A.1 From the above $L^{2}$ and $H^{1}$ estimates, we see that the time derivatives $\frac{d u}{d t}$ and $\frac{d w}{d t}$ are also uniformly bounded. Therefore, we obtain that $u$ and $w$ are in fact weakly continuous in time. Namely, $u, w \in C_{w}\left(H^{1} ;[0, T]\right)$ for all $T>0$, where the space $C_{w} \subset L^{\infty}$ here consists of all functions that are weakly continuous in time with values in $H^{1}$. In particular, the initial conditions are satisfied in the weak sense, i.e.,

$$
\lim _{t \rightarrow 0} \int_{\Omega_{i}} u(\cdot, t) \xi d x=\int_{\Omega_{i}} u(\cdot, 0) \xi d x
$$

for $i=1,2$ and any test function $\xi$ being $\phi$ or $\psi$. Moreover, by standard arguments, e.g. Constantin and Foias [1988], Foias et al. [2001], we have that the solution is actually analytic 
in time.

Remark A.2 In view of Sobolev embedding $H^{1} \hookrightarrow L^{\infty}$ in one dimensional space, we conclude that $u$ and $w$ are in fact uniformly bounded for all time.

Remark A.3 Now with the $H^{1}$ estimates available, we can repeat the above arguments but testing the system by $\Delta^{2} u$ and $\Delta^{2} w$ over $\Omega_{j}, j=1,2$, and obtain the $H^{2}$ boundedness of the solution (see e.g., Larios and Pei [2016]). Similarly, we have that the solution is in fact smooth. For simplicity, we omit the details here.

\section{References}

B.R. Anholt. Density dependence resolves the stream drift paradox. Ecology, 76:2235-2239, 1995.

Mary Ballyk, Le Dung, Don A. Jones, and Hal Smith. Effects of random motility on microbial growth and competition in a flow reactor. SIAM Journal of Applied Mathematics, 59(2): 573-596, 1998.

K.E. Bencala and R.A. Walters. Simulation of solute transport in a mountain pool-and-riffle stream: A transient storage model. Water Resources Research, 19(3):718-724, 1983.

B. Boldin. Persistence and spread of gastro-intestinal infections: the case of enterotoxigenic escherichia coli in piglets. Bulletin of Mathematical Biology, 70(7):2077-2101, 2007.

J.E. Byers and J.M. Pringle. Going against the flow: Retention, range limits and invasions in advective environments. Marine Ecology Progress Series, 313:27-41, 2006.

P. Constantin and C. Foias. Navier-Stokes Equations, Chicago Lectures in Mathematics. University of Chicago Press, Chicago, IL, 1988.

C. Foias, O. Manley, R. Rosa, and R. Temam. Navier-Stokes Equations and Turbulence, Encyclopedia of Mathematics and its Applications. Cambridge University Press, Cambridge, 2001.

A.E. Hershey, J. Pastor, B.J. Peterson, and G.W. Kling. Stable isotopes resolve the drift paradox for baetis mayflies in an arctic river. Ecology, 74:2315-2325, 1993.

Y. Jin and M.A. Lewis. Seasonal influence on population spread and persistence in streams: Critical domain size. SIAM Journal of Applied Mathematics, 71:1241-1262, 2011. 
Y. Jin, F.M. Hilker, P.M. Steffler, and M.A. Lewis. Seasonal invasion dynamics in a spatially heterogeneous river with fluctuating flows. Bulletin of Mathematical Biology, 76(7):1522-1565, 2014 .

A. Kolpas and R.M. Nisbet. Effects of demographic stochasticity on population persistence in advective media. Bulletin of Mathematical Biology, 72(5):1254-1270, 2010.

K.-Y. Lam, Y. Lou, and F. Lutscher. The emergence of range limits in advective environments. SIAM Journal of Applied Mathematics, 76(2):641-662, 2016.

A. Larios and Y. Pei. On the local well-posedness and a prodi-serrin type regularity criterion of the three-dimensional mhd-boussinesq system without thermal diffusion. arXiv, page https://arxiv.org/abs/1609.06002, 2016.

Y. Lou and F. Lutscher. Evolution of dispersal in open advective environments. Journal of Mathematical Biology, 69(6-7):1319-1342, 2014.

F. Lutscher and G. Seo. The effect of temporal variability on persistence conditions in rivers. Journal of Theoretical Biology, 283:53-59, 2011.

F. Lutscher, E. Pachepsky, and M.A. Lewis. The effect of dispersal patterns on stream populations. SIAM Review, 47(4):749-772, 2005.

F. Lutscher, M.A. Lewis, and E. McCauley. The effects of heterogeneity on population persistence and invasion in rivers. Bulletin of Mathematical Biology, 68(8):2129-2160, 2006.

F. Lutscher, E. McCauley, and M.A. Lewis. Spatial patterns and coexistence mechanisms in rivers. Theoretical Population Biology, 71(3):267-277, 2007.

F. Lutscher, R. Nisbet, and E. Pachepsky. Population persistence in the face of advection. Theor. Ecol., 3:271-284, 2010.

K. Müller. Investigations on the organic drift in north swedish streams. Technical Report 34, Institute of Freshwater Research, Drottningholm, 1954.

K. Müller. The colonization cycle of freshwater insects. Oecologica, 53:202-207, 1982.

E. Pachepsky, F. Lutscher, R. Nisbet, and M. A. Lewis. Persistence, spread and the drift paradox. Theoretical Population Biology, 67:61-73, 2005.

V.B. Pasour and S.P. Ellner. Computational and analytic perspectives on the drift paradox. SIAM Journal of Applied Dynamical Systems, 9:333-356, 2010.

J.M. Pringle, F. Lutscher, and E. Glick. Going against the flow: the effect of non-gaussian dispersal kernels and reproduction over multiple generations. Marine Ecology Progress Series, 337:13-17, 2009. 
J.M. Ramirez. Population persistence under advection-diffusion in river networks. Journal of Mathematical Biology, 65(5):919-942, 2012.

Y. Samia and F. Lutscher. Persistence probabilities for stream populations. Bulletin of Mathematical Biology, 74:1629-1650, 2012.

J. Sarhad, R. Carlson, and K. Anderson. Population persistence in river networks. Journal of Mathematical Biology, 69:401-448, 2014.

G. Seo and F. Lutscher. Spread rates under temporal variability: calculation and application to biological invasions. Mathematical Models and Methods in Applied Sciences, 2011.

N. Shigesada, K. Kawasaki, and E. Teramoto. Traveling periodic waves in heterogeneous environments. Theoretical Population Biology, 30:143-160, 1986.

D.C. Speirs and W.S.C. Gurney. Population persistence in rivers and estuaries. Ecology, 82(5): 1219-1237, 2001.

R. Temam. Navier-Stokes Equations, Theory and Numerical Analysis, Reprint of the 1984 edition. AMS Chelsea Publishing, Providence, RI, 2001.

O. Vasilyeva, F. Lutscher, and M.A. Lewis. Analysis of spread and persistence for stream insects with winged adult stages. Joural of Mathematical Biology, 72(4):851-875, 2016.

H. F. Weinberger. Long-time behavior of a class of biological models. SIAM Journal of Mathematical Analysis, 13:353-396, 1982.

H. F. Weinberger. On spreading speeds and traveling waves for growth and migration models in a periodic habitat. Journal of Mathematical Biology, 45:511-548, 2002. 